REVIEW ARTICLE

\title{
Doppler in Myometrial Lesions
}

\author{
Sonal Panchal ${ }^{1}$, Chaitanya Nagori ${ }^{2}$
}

\begin{abstract}
Myometrium is the chief bulk-forming layer of the uterus. Abnormalities of the myometrium may lead to abnormal peristalsis and abnormalities of circulation, and both may lead to dysfunctional uterine bleeding (DUB) and/or infertility. But, many of these are detected only as incidental finding. Assessment of myometrium, therefore, requires a systematic approach, and this has been defined by morphological uterus sonographic assessment (MUSA). This helps correct localization of the lesion and also decides the surgical approach. B mode as well as Doppler plays an important role in confident differential diagnosis of the common myometrial lesions: adenomyosis and fibroids.

Keywords: Adenomyosis, Fibroid, Morphological uterus sonographic assessment.

Donald School Journal of Ultrasound in Obstetrics and Gynecology (2019): 10.5005/jp-journals-10009-1594
\end{abstract}

\section{INTRODUCTION}

Myometrium - the muscle layer of the uterus-forms the major bulk of the uterus mass. It surrounds the endometrial cavity and is surrounded by the serosa. The inner layer of the myometrium is known as endometriomyometrial junctional zone (JZ). This layer is structurally and functionally different from the rest of the myometrium. It is responsible for the initiation of the peristalsis in the endometrium. Myometrium is normally homogeneously hypoechoic, and the serosal surface is smooth and regular. Distortion of the shape or change in echogenicity suggests myometrial lesion.

\section{Scan Routes and Modalities}

Myometrial evaluation on ultrasound can be done by transabdominal route or transvaginal route. Both are supplementary to each other. Transabdominal scan is used to define the extent and margins of the myometrium. It is especially required in cases with large uterus or in cases in which due to space-occupying lesions like fibroid, the uterus is pushed upward and its superior margin cannot be accessed by transvaginal scan. Whereas the detailed morphology of the myometrium can be better studied by transvaginal scan due to proximity of probe to the uterus and better resolution due to high-frequency probe. Uterus is evaluated in sagittal and transverse planes on B-mode ultrasound. Coronal plane of the uterus may be best evaluated by 3D ultrasound. For assessment of the vascular changes, Doppler is the modality of choice.

\section{Assessment of the Uterus on Transvaginal SCAN}

Patient is first asked to empty the bladder and is placed in lithotomy position on the gynec couch in the same way as for per speculum or per vaginal examination. Ultrasound jelly is put on the head of the transvaginal probe and then the probe is covered with the condom, not to allow any air between the probe and the condom. A small amount of jelly is then placed over the condom on the probe head, and the probe is gently slid into the patient's vagina. In case of difficulty in introduction or patient's resistance to introduction, she is advised to take deep long breaths with open mouth, i.e., deep inspirations and long complete expirations. Counseling the patient before examination and explaining the whole procedure
1,2Dr Nagori's Institute for Infertility and IVF, Ahmedabad, Gujarat, India Corresponding Author: Sonal Panchal, Dr Nagori's Institute for Infertility and IVF, Ahmedabad, Gujarat, India, Phone: +91 9824050911, e-mail: sonalyogesh@yahoo.com

How to cite this article: Panchal S, Nagori C. Doppler in Myometrial Lesions. Donald School J Ultrasound Obstet Gynecol 2019;13(3):113-128. Source of support: Nil

Conflict of interest: None

and adequate privacy help eliminate the anxiety and resistance. Probe is introduced in the longitudinal position with indicator on the probe facing the patient's anterior aspect (Fig. 1).

This indicator on the probe matches the logo on the screen. This means that if the indicator on the screen is on the left side of the screen, the structures anteriorly placed are on the left side of the screen (Fig. 2). Longitudinal axis of the patient normally corresponds to the long axis of the uterus. That means the uterus must be seen in the sagittal plane on this view. It may be anteverted or retroverted. When anteverted, the uterine fundus will be directed toward the indicator on the screen and urinary bladder will also be seen on the same side of the screen, and when retroverted fundus will be on the opposite side of the indicator and the urinary bladder (Fig. 3). If the uterus is not seen in this view, it means that the uterus is deviated toward one side. When it is deviated, it would indicate that either it is pulled on the side of the pathology like adhesions or is pushed away by the pathology, which is on the opposite side, say a mass.

Once the midsagittal plane of the uterus is seen, span the probe from one side to another, without rotating it, for the anatomical survey of the uterus in longitudinal axis. The spanning should extend from one side of the uterus across the midsagittal plane to the other side, till the uterus goes out of vision. Then the probe is realigned in the midsagittal plane of the uterus and rotated $90^{\circ}$ anticlockwise. This maneuver will show transverse view of the uterus with the right side of the patient on right side of the screen (Fig. 4). In this transverse position also, the whole uterus is evaluated from the fundus to the cervix by tilting the probe up and down in the vagina. This survey should give a complete idea about the anatomy of the uterus and the exact location of any pathology. Any localized lesion if seen in the uterus, its location in all three planes (sagittal, axial, and coronal) must be confirmed. This understanding of the orientation of the image and the uterus and the probe movement 


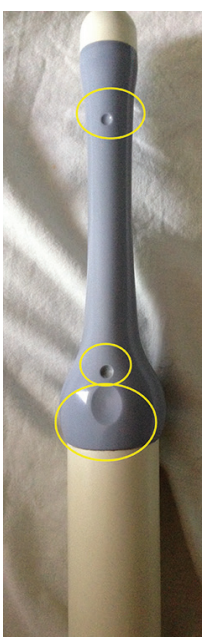

Fig. 1: Transvaginal volume probe showing the markers
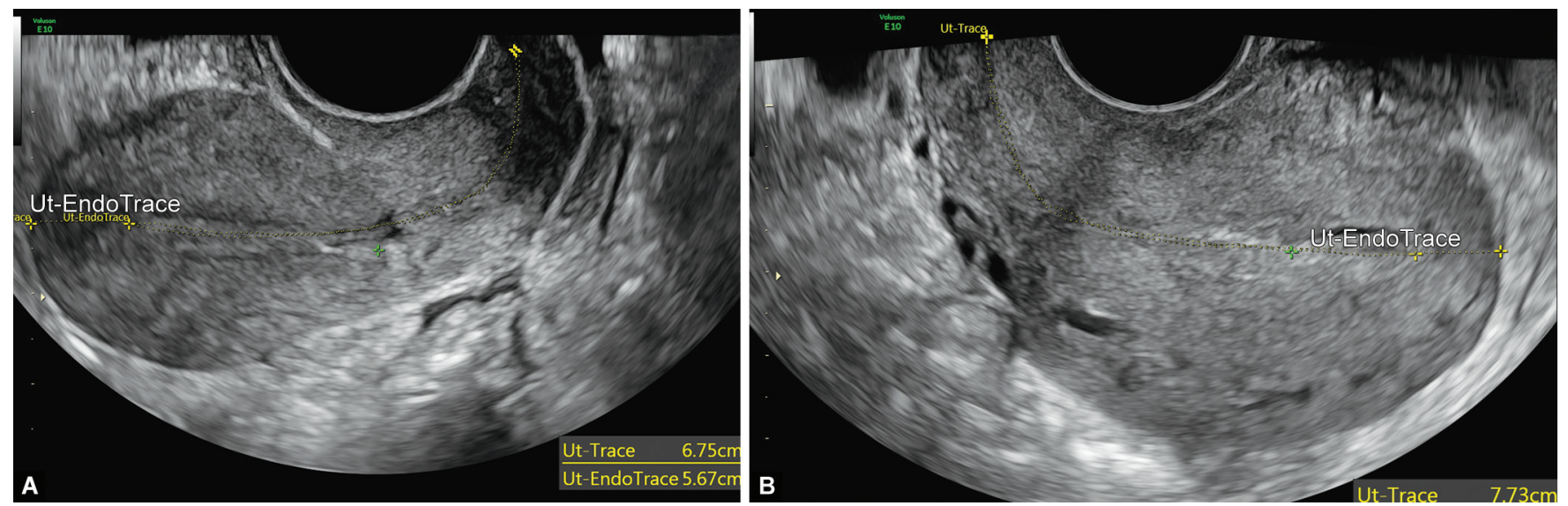

Figs $3 \mathrm{~A}$ and B: (A) Midsagittal section of the uterus showing anteverted anteflexed uterus; (B) Midsagittal section of the uterus showing retroverted retroflexed uterus

is essential to correctly diagnose the location of the lesions in the myometrium.

Myometrial lesions like fibroids and adenomyosis are among the most common uterine pathologies and still these are the ones that bring most surprises on surgery as these are most inaccurately reported. The inaccuracy is because of random terms used for the description of ultrasound image and nonspecific terms used for localization. It is for this that a consensus was formed to describe the myometrial appearance or lesions and was termed as morphological uterus sonographic assessment (MUSA).

\section{MUSA $^{1}$}

It accurately describes the measurement techniques, specifies on the terms used for various ultrasound pictures, clearly defines the reporting formats, and also helps to identify reliable characters of benignity.

\section{Measurements}

The length of the uterus is measured as length of the three different segments. Fundal length is the distance from the fundal serosa to the fundal end of the endometrial cavity, in the line that is in continuity with the endometrial cavity. The second segment is the length of the endometrial cavity measured as a trace from fundal

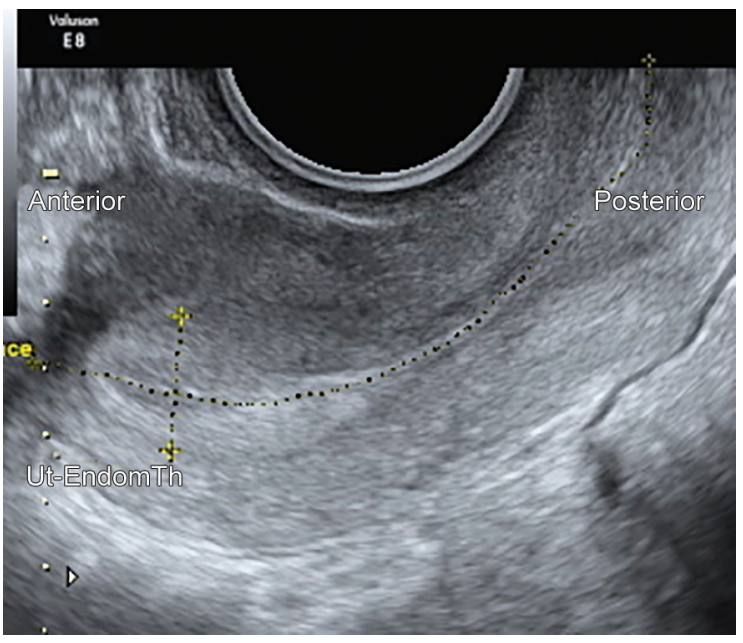

Fig. 2: Midsagittal section of the uterus on B mode on transvaginal scan indicating anterior and posterior aspect of the patient tip of the endometrium to the internal os. The third segment is the cervical length, length from the internal os to external os, measured as a trace (Fig. 5).

The anteroposterior (AP) diameter is measured in this same midsagittal plane as the longest diameter on the uterus body, perpendicular to the endometrial cavity (Fig. 6). The probe is then rotated for the transverse section $\left(90^{\circ}\right.$ anticlockwise) to achieve the transverse section of the uterus. The broadest distance on this section, side to side is the transverse diameter (Fig. 7). The uterine volume is calculated as total length $\times$ width $\times$ AP diameter $\times 0.523$. The thickness of the anterior and posterior myometrial walls may be measured separately when these appear unequal or asymmetrical. This is measured as the longest distance from outer margin of endometrium to the serosa, perpendicular to the central line of the endometrium, both anteriorly and posteriorly (Fig. 8). The endometrial thickness is also measured on this same plane, at the level where the endometrium is the thickest and it is measured from outer margin of the endometrium to outer margin of the endometrium, (anterior and posterior), perpendicular to the central line of the endometrium (Fig. 6). Junctional zone thickness is the measurement of the thickness of the hypoechoic zone in the most proximity of the outer hyperechoic margin of the endometrium, perpendicular to the endometrial outer margin and if regular it is 


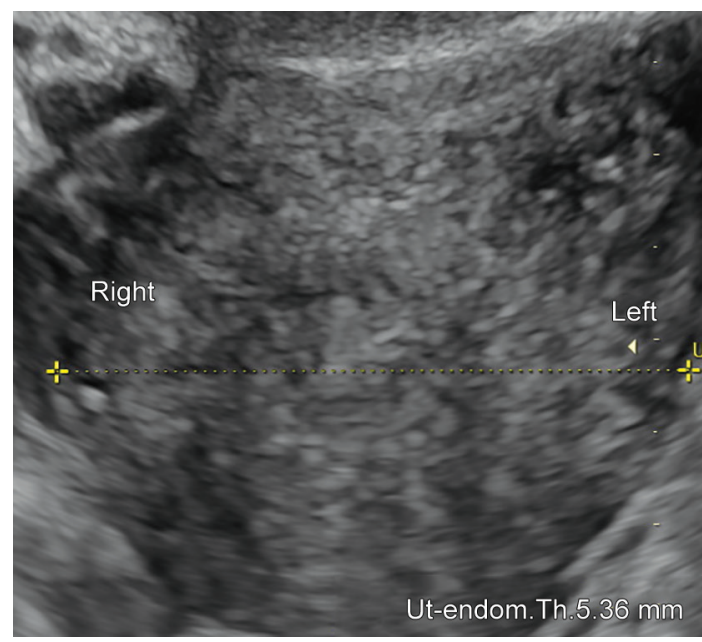

Fig. 4: Transverse section of the uterus with right and left side of the patient marked on the image

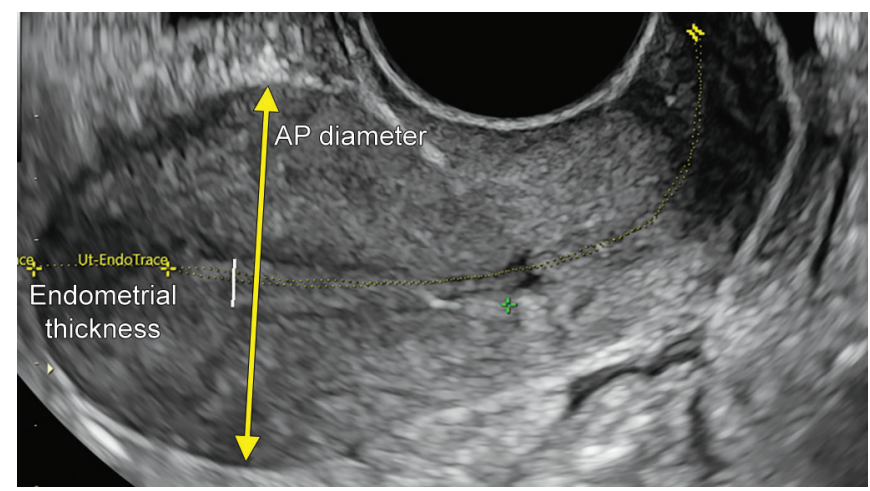

Fig. 6: AP diameter and endometrial thickness of the uterus on midsagittal section of the uterus, measured according to MUSA

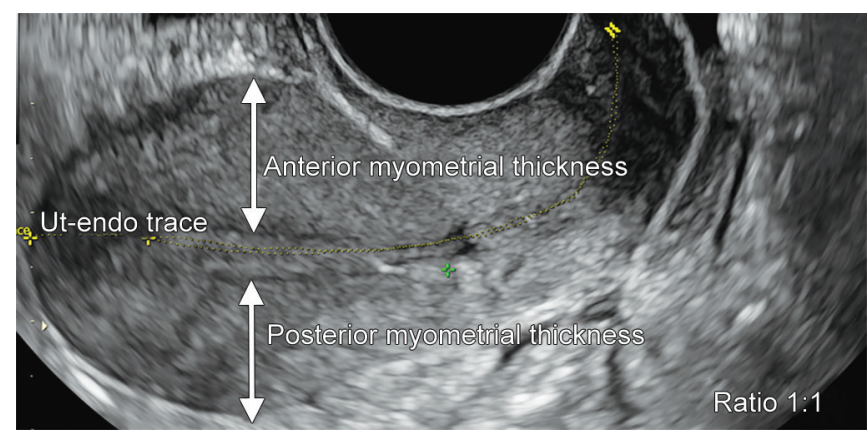

Fig. 8: Anterior and posterior myometrial diameter measured on midsagittal section of the uterus, measured according to MUSA

measured as shown in the figure (Fig. 9). Junctional zone is best assessed on 3D ultrasound with volume contrast imaging ( $\mathrm{VCl}$ ) (Fig. 10). If it is irregular, the maximum and the minimum thickness of the JZ is measured and is also important to mention the location and extent of the irregularity.

\section{Description: Terminology Used According to MUSA}

Junctional zone qualitatively is described as regular, irregular (Fig. 11), interrupted (Fig. 12) or inaccessible (Fig. 13). The interruption of the JZ may be because of cystic areas, hyperechogenic dots, buds, or hyperechoic lines (Fig. 14). Junctional zone may be interrupted

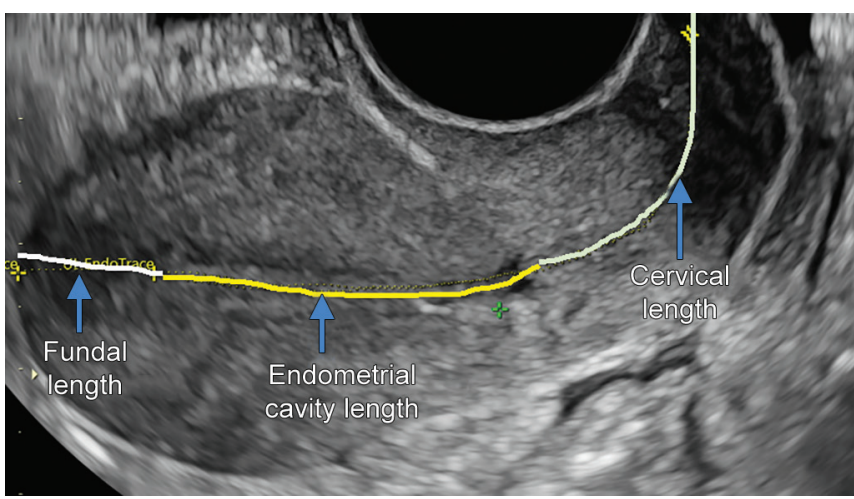

Fig. 5: Longitudinal measurements of the uterus on midsagittal section of the uterus, measured according to MUSA

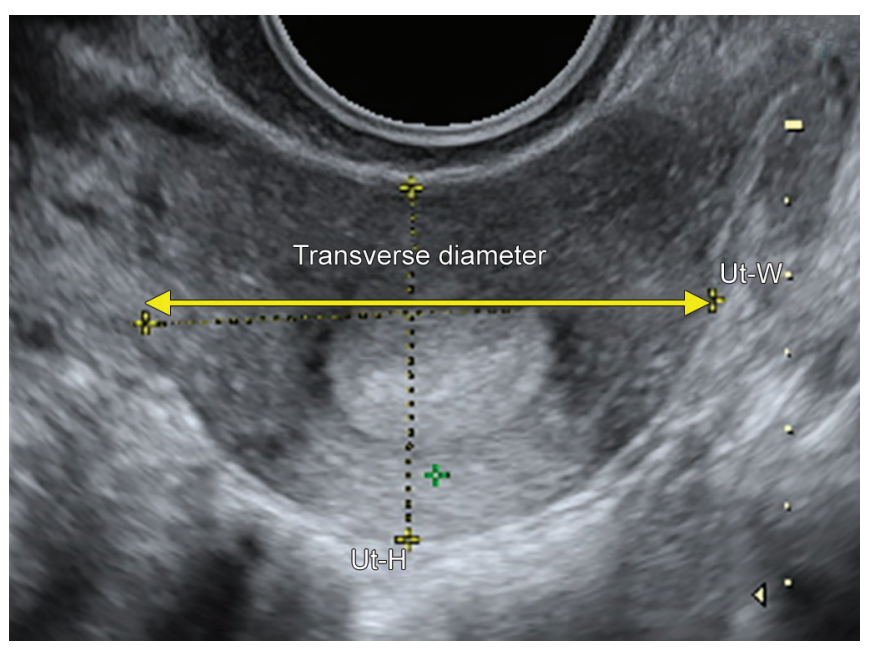

Fig. 7: Transverse diameter of the uterus on transverse section of the uterus, measured according to MUSA

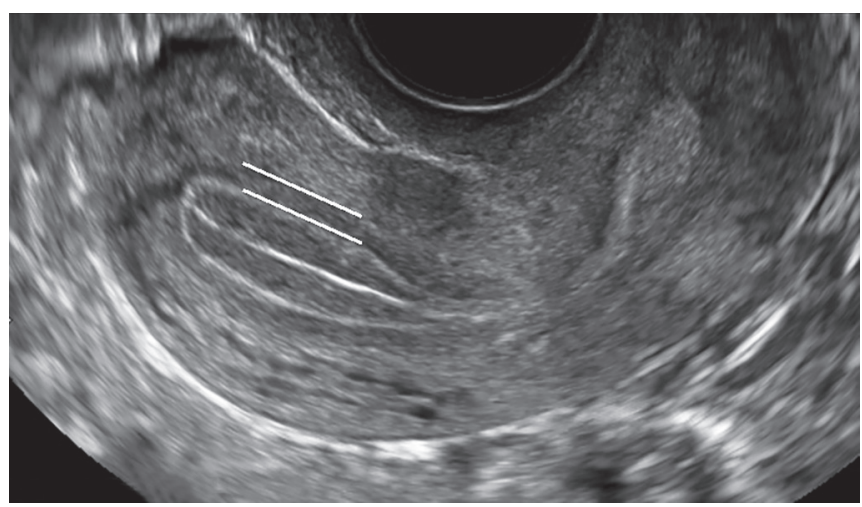

Fig. 9: Junctional zone measurement of the uterus on midsagittal section of the uterus, measured according to MUSA

due to focal lesions/contractions. When the entire JZ cannot be identified, it is nonaccessible. But it may also be mentioned as inaccessible when it may not be clearly seen due to poor visibility, in obese patients or when uterus is deep.

If it is irregular, its thickness is measured at the thickest area of the JZ (Fig. 15). When JZ is interrupted or irregular, severity of irregularity is documented as a ratio of thickness of the thickest part of the JZ and thickness of the entire myometrial thickness, measured at the same level, in the same image, and in the same 


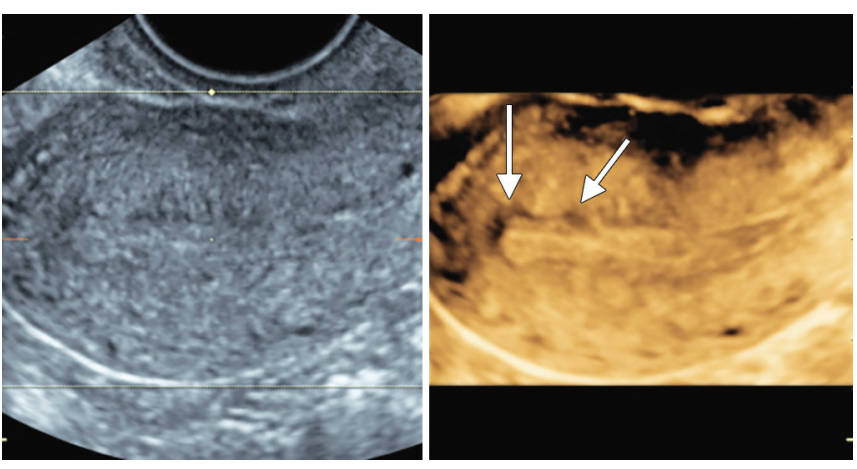

Fig. 10: $B$ mode and $\mathrm{VCl} A$ of the midsagittal section of the uterus, demonstrating clear delineation of the $\mathrm{JZ}$ on $\mathrm{VCl} A$

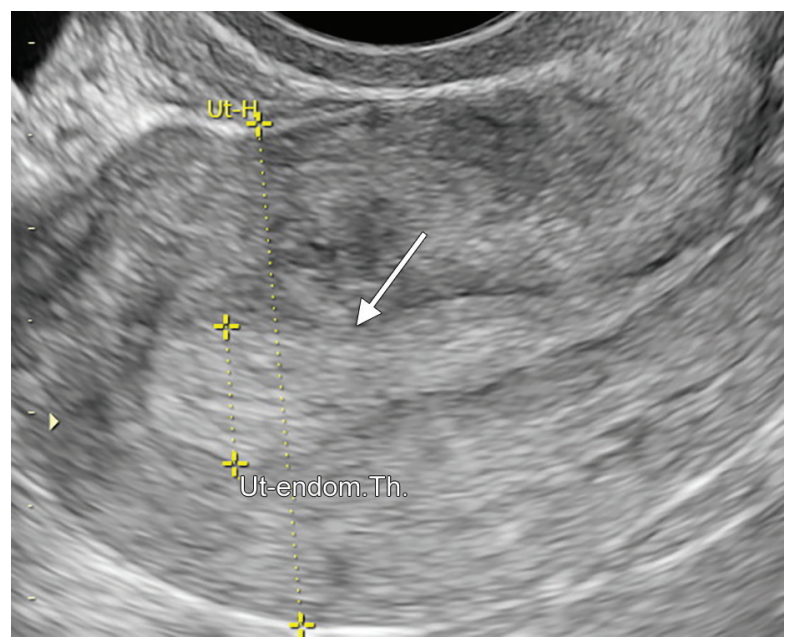

Fig. 12: Transvaginal ultrasound B mode showing anteverted anteflexed uterus, midsagittal section of the uterus with interrupted endometriomyometrial junction on the anterior aspect

plane (Fig. 16). Magnitude of a JZ irregularity is expressed as the difference between the maximum and minimum JZ thickness: $\left(J Z_{\text {dif }}\right)=J Z_{\text {max }}-J Z_{\text {min }}$ (Fig. 15).

It is essential to mention that the irregularity/interruption involves anterior wall, posterior wall, right lateral wall, left lateral walls, or fundus. Extent of the irregularity can be subjectively described as $<50 \%$ or $>50 \%$, for the uterus as a whole or for each location.

\section{Myometrial Description}

Myometrial texture is described as homogeneous or heterogeneous in echotexture/echogenicity (Fig. 17). Heterogenicity may be due to cystic areas, hyperechogenicities, or shadowing (Fig. 18). Heterogenicity may be localized (one/more areas) (Fig. 19A) or diffuse (Fig. 19B and Flowchart 1).

When the lesion is localized, it is essential to mention whether it is well-defined (Fig. 20) or ill-defined (Fig. 19A). Its location is described as anterior, posterior, fundal, right lateral, or left lateral and it involves upper, mid, or lower body or cervix. If the lesion is diffused, it is described as a global heterogenicity/global involvement of the uterus by the lesion. The size of the lesion is measured as three orthogonal diameters. The longest is measured in the longest plane, the AP diameter is the longest diameter perpendicular to this and on a transverse plane, that is achieved

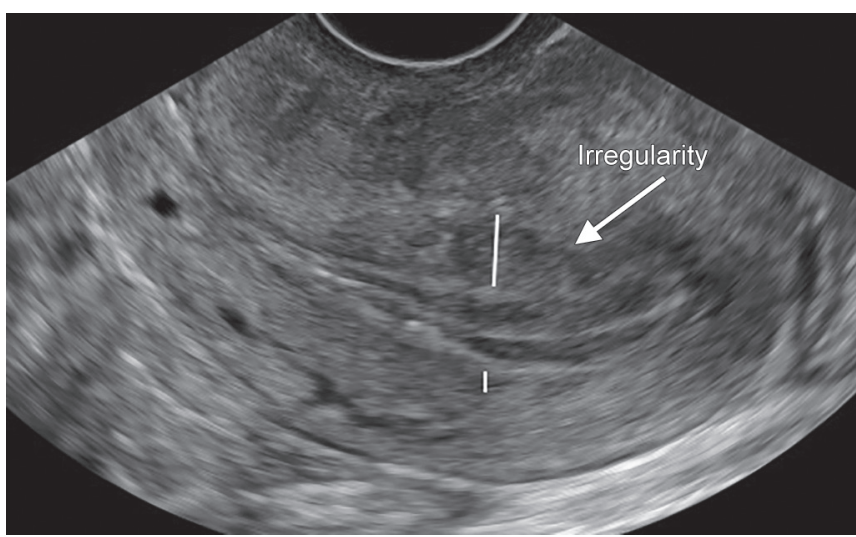

Fig. 11:Transvaginal ultrasound B mode showing retroverted retroflexed uterus, midsagittal section of the uterus with irregular endometriomyometrial junction on the posterior aspect

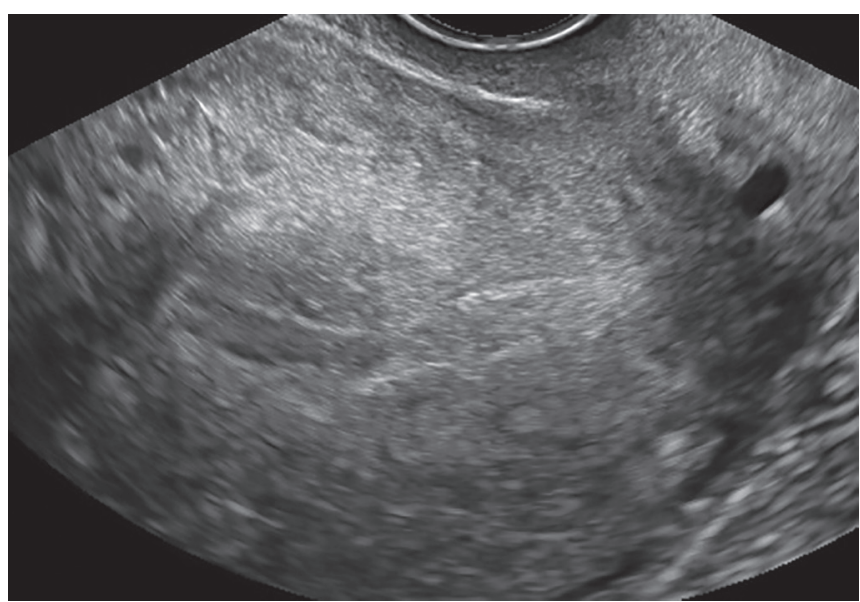

Fig. 13: Transvaginal ultrasound B mode showing anteverted anteflexed uterus, midsagittal section of the uterus with inaccessible endometriomyometrial junction on the anterior aspect

by only $90^{\circ}$ rotation of the probe (Fig. 21). The site of the lesion is described as anterior, posterior, right or left lateral or fundal and then whether it is involving upper, middle, or lower part of the corpus or cervix is mentioned. Since myometrium is a thick layer of the uterus, most lesions will not involve its entire thickness. Therefore, it is essential to mention how much thickness of the myometrium is affected by the lesion and also whether peripheral layer of the myometrium or central layer of the myometrium is involved. These lesions are therefore classified according to PALMCOEIN classification. ${ }^{2}$ For the exact localization of the lesion and also to get the guidance about the route of surgery, minimum distance of the lesion from endometrium (inner lesion-free margin) or minimum distance from serosa (outer lesion-free margin) must also be mentioned (Fig. 22).

Description of the lesion includes its shape (round, oval, lobulated, irregular), rim/margins (ill-defined, hypoechoic, and hyperechoic), and its echogenicity (homogeneous/heterogeneous). Most lesions in the myometrium cast an acoustic shadow and that must also be mentioned. These may be fan-shaped (alternate hyper- and hypoechoic bands) (Fig. 23A) with or without edge shadows (Fig. 23B). Amount of shadowing is described as slight, moderate, or strong. The hyper-/hypoechogenicity of the lesion is described as follows: 


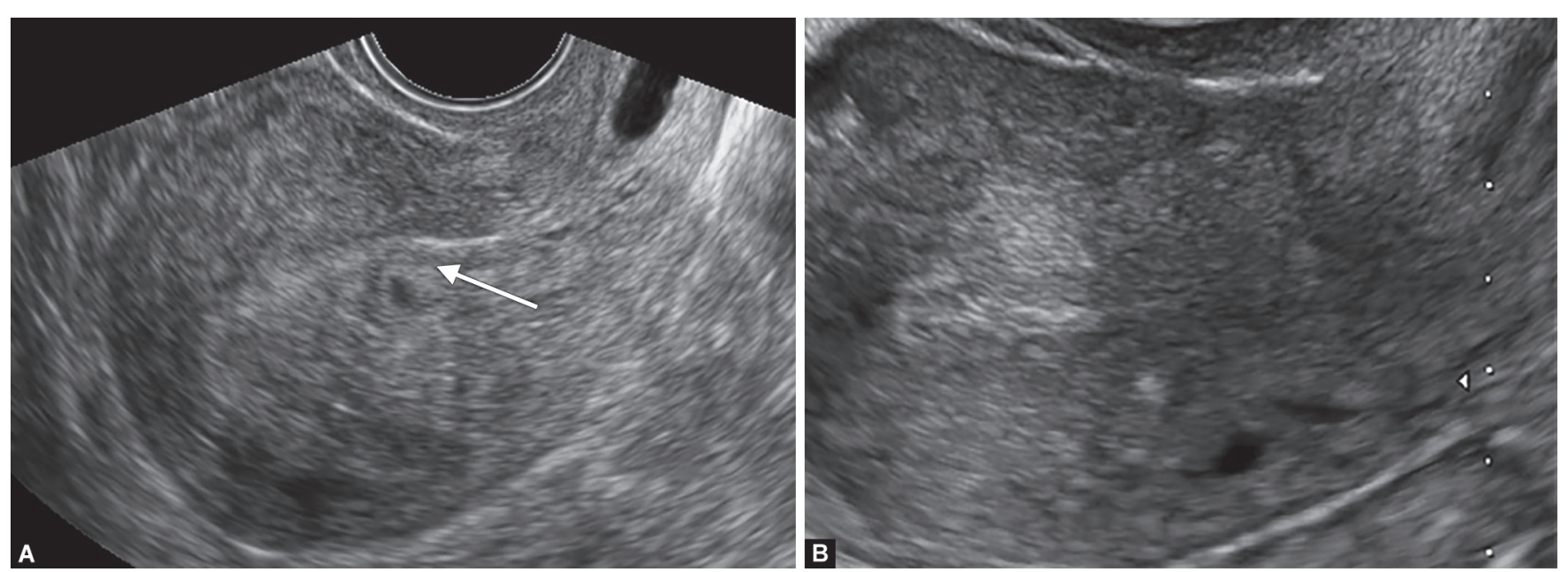

Figs 14A and B: (A) Transvaginal ultrasound B mode showing anteverted anteflexed uterus with interruption of the JZ because of cystic areas; (B) Transvaginal ultrasound B mode showing anteverted anteflexed uterus with interruption of the JZ because of hyperechoic islands

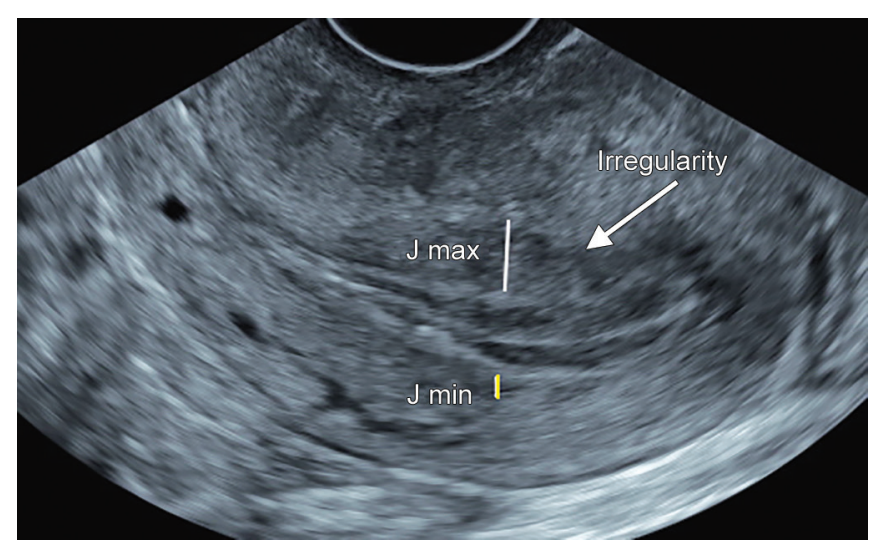

Fig. 15:Transvaginal ultrasound B mode showing retroverted retroflexed uterus with irregularity of the JZ. The white line shows maximum thickness, and yellow line shows minimum thickness of the JZ

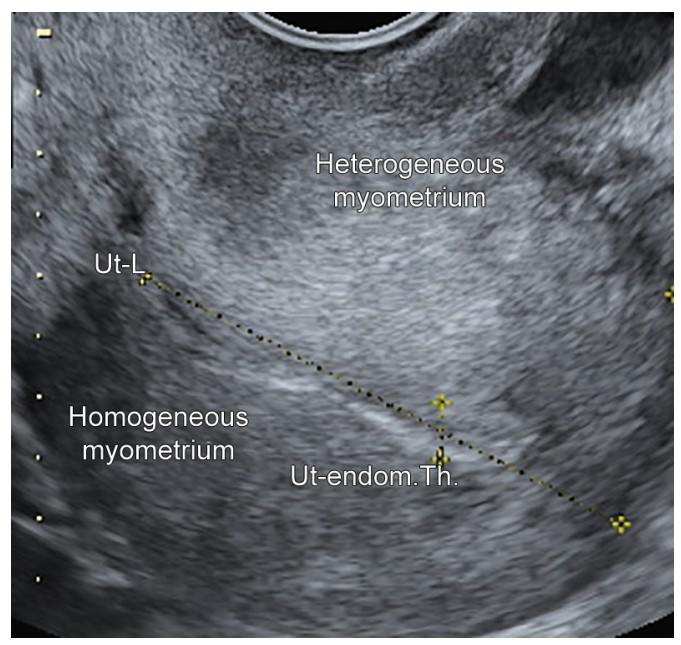

Fig. 17: Retroverted retroflexed uterus on B mode, transvaginal ultrasound showing heterogeneous and thick posterior myometrium and homogeneous normal anterior myometrium

Level of echogenicity: It is compared to that of normal myometrium.

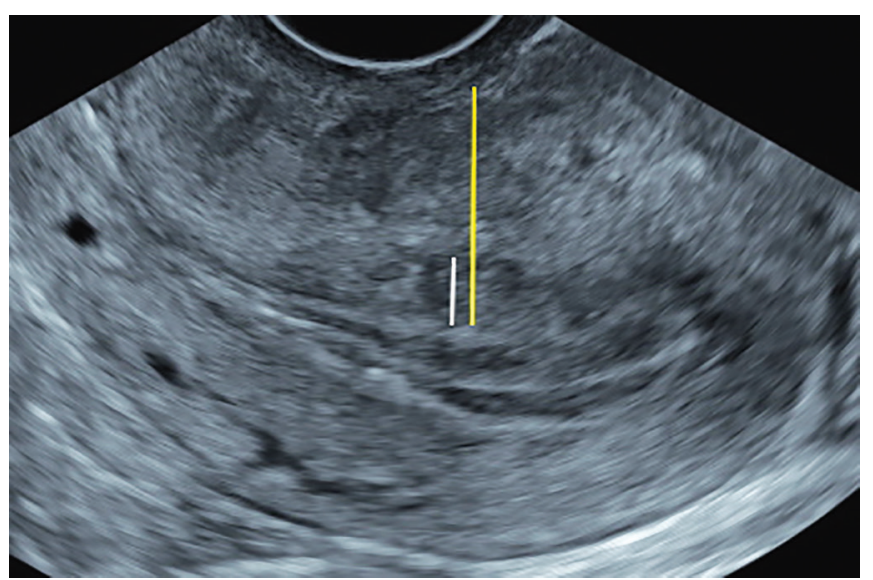

Fig. 16: Transvaginal ultrasound B mode showing retroverted retroflexed uterus with irregularity of the JZ. The white line shows maximum thickness of the JZ, and yellow line shows thickness of the posterior myometrium

- Very hypoechogenic (--)

- Hypoechogenic (-)

- Isoechogenic

- Hyperechogenic (+)

- Very hypogenic $(++)$

- Detailed description on the echogenicity is required.

When the lesion is diffused, penetration of the diffused lesions is described as a ratio between the maximum thickness of the lesion to the total uterine wall thickness and extent as greater than or less than $50 \%$ of the total uterus body length. Anterior/ posterior/lateral/fundal wall involvement is to be mentioned like localized lesion.

\section{Myometrial Heterogenicities}

Myometrial echogenicity is called to be heterogeneous either in presence of localized lesion or in presence of myometrial cysts (Fig. 18A), hyperechoic islands (Fig. 24), and hyperechogenic lines (Fig. 18B)/dots. These are most often found in adenomyosis but may be seen in certain chronic inflammations, postsurgical, vascular abnormalities, etc. 

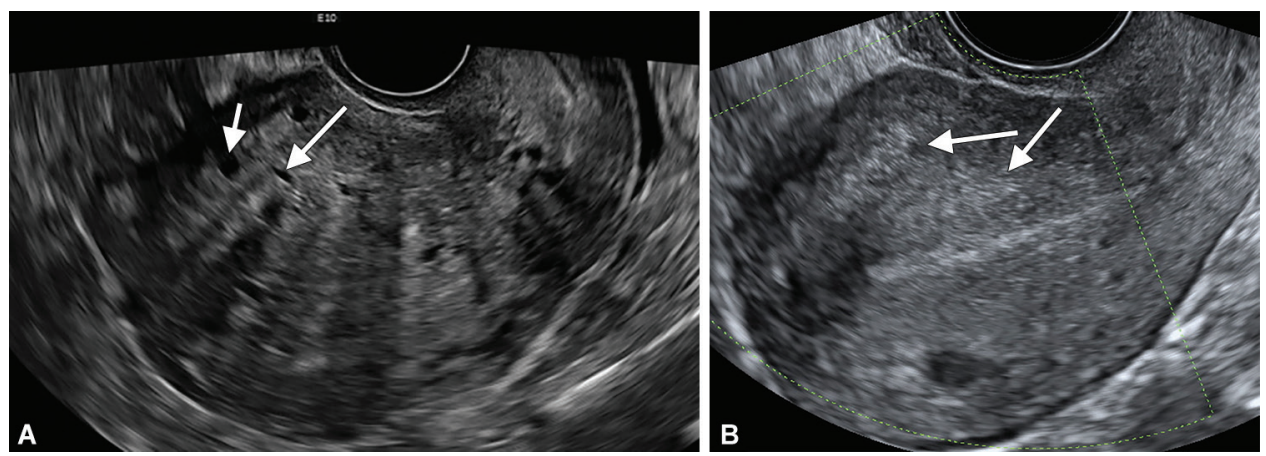

Figs 18A and B: (A) B-mode ultrasound image of the uterus showing anechoic cystic areas of myometrial cysts marked by arrows; (B) Hyperechoic lines marked by arrows
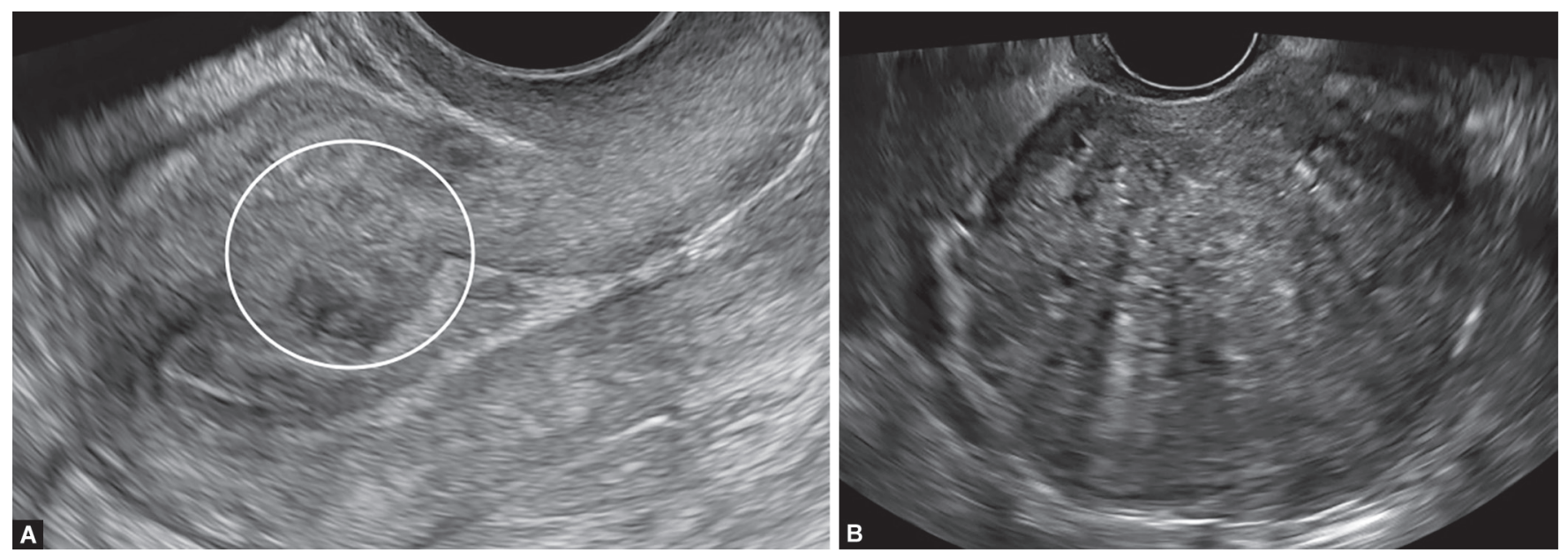

Figs 19A and B: (A) Anteverted anteflexed uterus on B mode on transvaginal scan with localized area of heterogeneous myometrium as marked by the circle, though the margins are ill defined; (B) Generalized heterogenicity of the myometrium

Flowchart 1: Assessment for myometrium

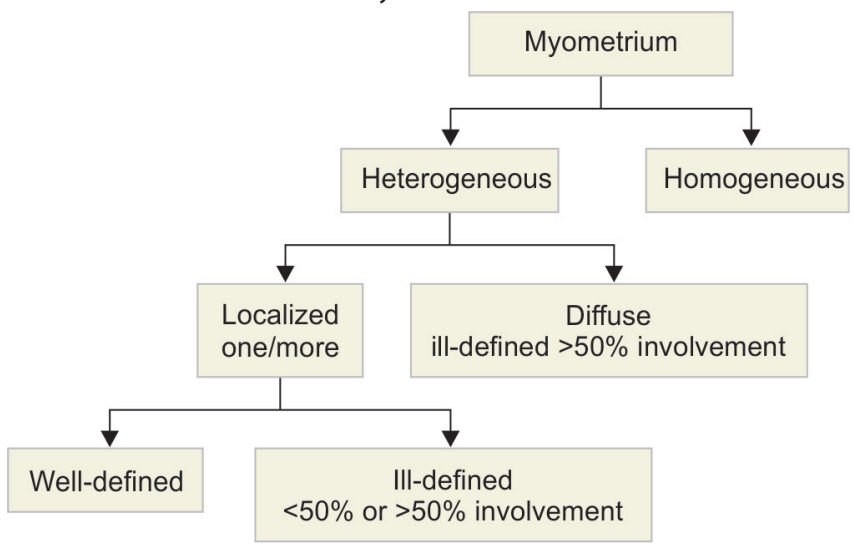

\section{Myometrial Cysts}

These are anechoic well-defined, mostly rounded lesions. The contents may be anechoic and may have low-level echogenicities, ground-glass echogenicities, or mixed echogenicities. One must mention number of cysts, locations, and largest diameters of the largest cyst. When these are distributed widely, one needs to mention about the walls that are involved, whether the upper, middle, or lower third of the body of uteurs is onvolved and whether it is involving greater than or less than $50 \%$ of the myometrium. If multiple small cysts aggregate, anechoic lacunae are formed that may be irregular in shape.

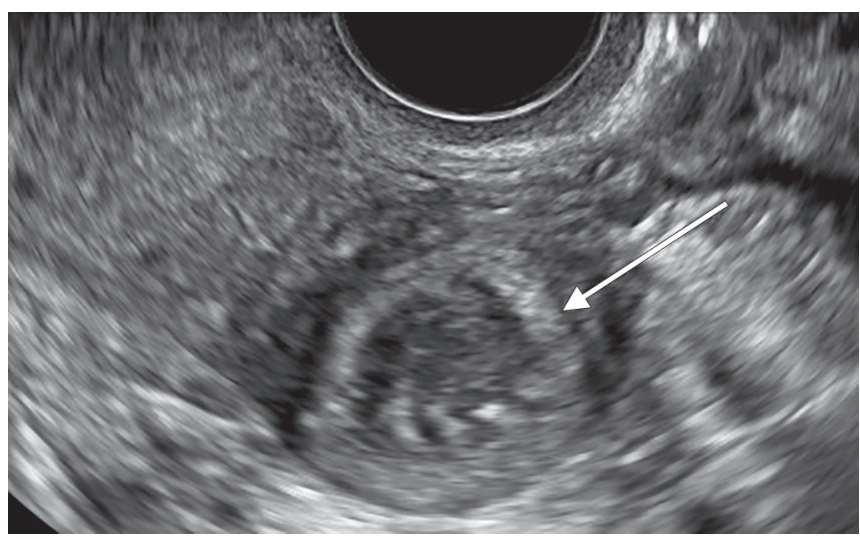

Fig. 20: A well-defined localized lesion is seen in the posterior wall of the uterus as marked by the arrow

\section{Hyperechoic Islands}

These are regular, irregular, or ill-defined hyperechoic areas. Same as for cysts, it is essential to mention the number of hyperechoic islands, size of the largest, and location of each. These are most commonly due to endometrial growth in the myometrium and are seen in patients with adenomyosis.

\section{Hyperechoic Lines and Buds}

Hyperechoic lines and buds are seen leading to irregularity or interruption of the JZ. These hyperechoic lines and buds are 

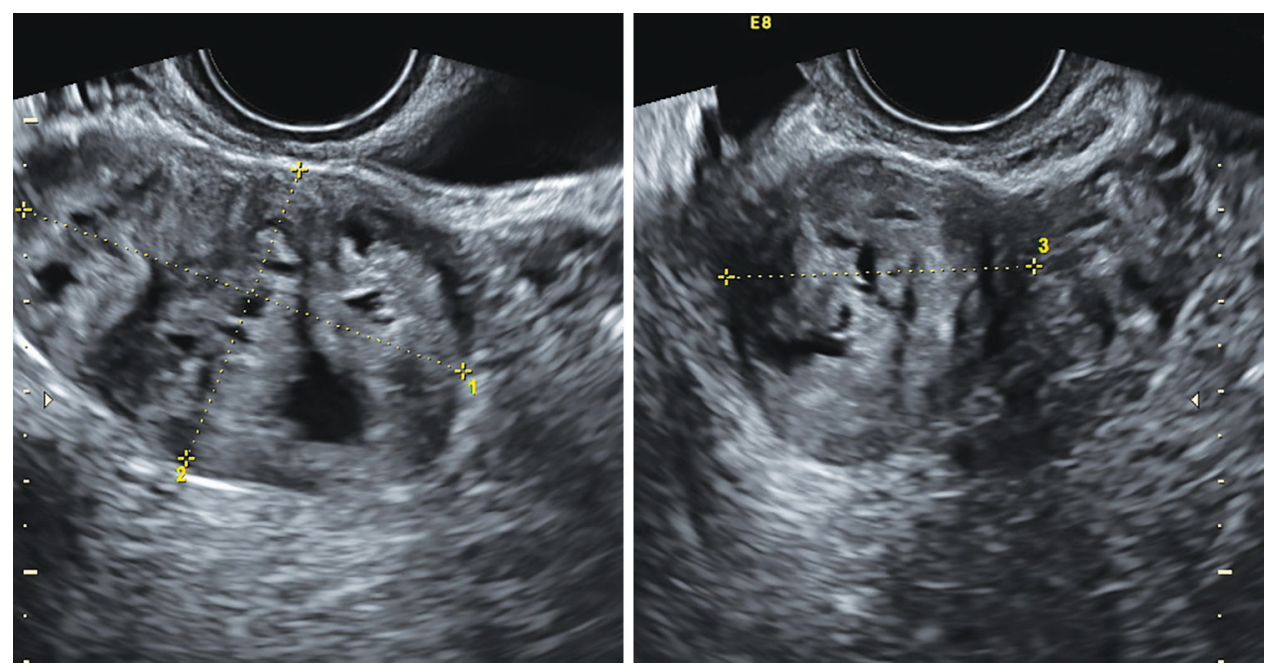

Fig. 21: Measurements of the localized myometrial lesion (fibroid), the long and the AP diameter measured on long section and transverse diameter on transverse section of the lesion

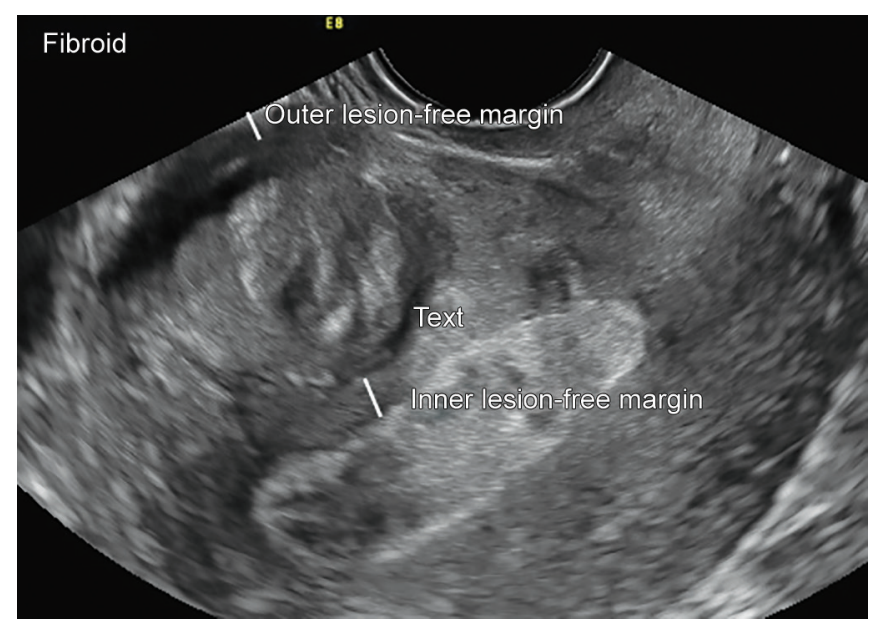

Fig. 22: Transvaginal ultrasound B mode showing anteverted anteflexed uterus with anterior intramural fibroid showing outer and inner lesionfree margins

different from hyperechoic spots. Whether these lines and buds are in continuity with the endometrium and whether they are perpendicular to endometrium or not needs to be mentioned. These lines and buds affect anterior, posterior, right or left lateral or fundal JZ and what is the extent of the irregular or interrupted endometrium needs to be mentioned. If these are countable, their number needs to be mentioned.

The vascularity study gives more information on these lesions, but it is very essential to evaluate the myometrium on B mode and adequately analyze it before vascularity studies are done.

\section{Doppler Studies}

The distribution of the vascularity is described as uniform if the entire lesion has uniformly distributed vascularity or nonuniform if it shows heterogeneous distribution of blood vessels in the lesion (Fig. 25). According to MUSA, the vascularity of the myometrial lesions can be scored as following: 1-no color, 2-minimal vascularity, 3-moderate vascularity, and 4-abundant vascularity (Fig. 26). Scoring is done, taking into consideration the most vascular area of a lesion. In lesions that show heterogeneous vascular distribution, do mention what percentage of the solid component is vascular. Commonly found myometrial lesions may show circumferential (Fig. 27A), intralesional, both (Fig. 27B), or translesional (Fig. 27C) vascularity. Circumferential is a peripheral vascularity of a lesion (like seen in a fibroid). The vessels that are seen in the lesion are intralesional vessels. Lesions like degenerated fibroids may show both circumferential and intralesional vascularity. Lesions like adenomyosis will show vessels traversing perpendicular to the uterine cavity/serosa crossing the lesion. These are described as translesional vessels.
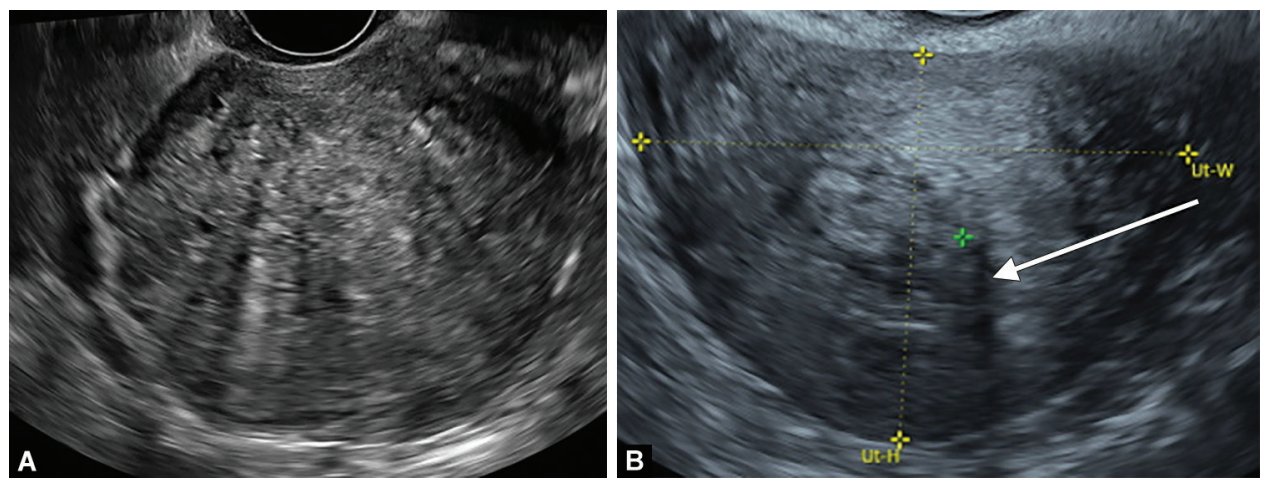

Figs 23A and B: (A) Fan-shaped acoustic shadowing; (B) Edge shadows as indicated by the arrow 
Further description about the vessels that can be studied by Doppler is the number of vessels (single/multiple) and diameter of vessels as compared to normal myometrial vessels (large/small). Whether all vessels in the lesion have an equal diameter (Fig. 28A) or they vary in diameter needs to be mentioned. It is essential to specifically mention whether the variability in diameter is seen in individual vessels also or is seen in different vessels. This may be reported as abnormal tortuous vessels, irregular caliber, when individual vessel shows variability in caliber (Fig. 28B).

The branching pattern of the vessels is especially different in benign and malignant lesions and so documenting the regular/ irregular branching pattern is important. Branching pattern of vessels in malignant lesions is typically described as lack of

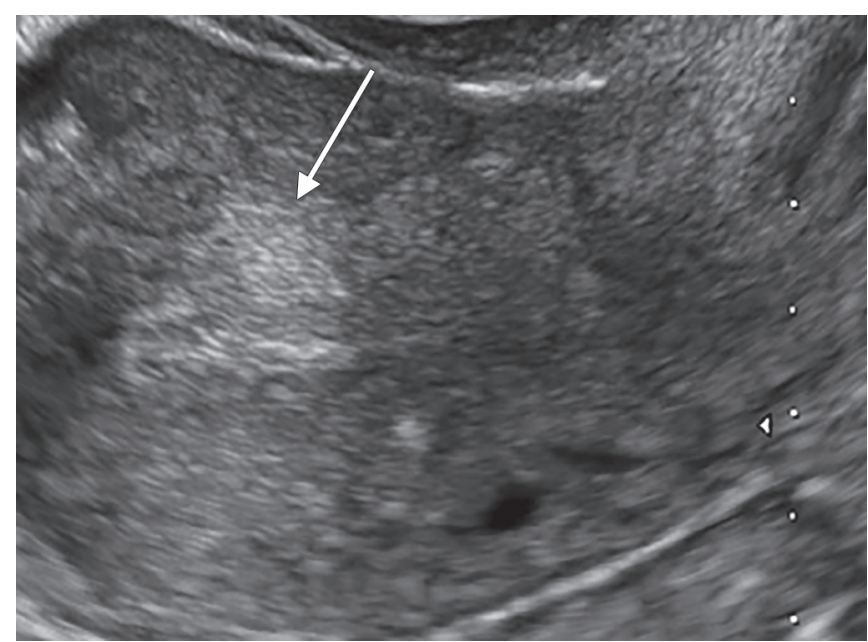

Fig. 24: Transvaginal ultrasound B mode showing anteverted anteflexed uterus with hyperechoic localized area-hyperechoic island as marked by the arrow
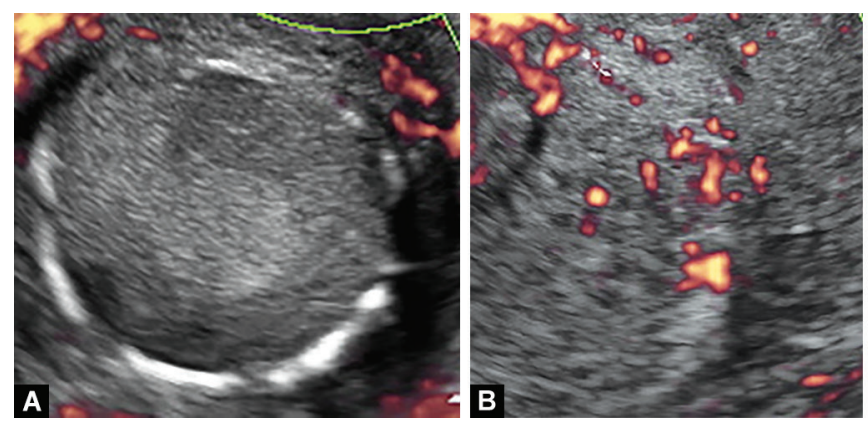

hierarchy in branching, varying branching angles, and chaotic vessel pattern.

The reporting should include:

- Size

- Symmetry of myometrial walls

- Echogenicity

- Junctional zone-regular, irregular, ill-defined

- Extent and magnitude of irregularity

- Lesion: well-defined/ill-defined, number, location, size, maximum diameter, echogenicity of the lesion (cysts, hyperechoic islands, buds, lines, spots, shadowing)

- Mapping of lesions

- Vascularity - pattern, score, branching pattern, number of vessels, diameter

- If the lesion is present, is it relevant to the symptoms.

Keeping into consideration the descriptive terms discussed, let us now look into the ultrasound presentation of common myometrial lesions, with special consideration on their Doppler findings.
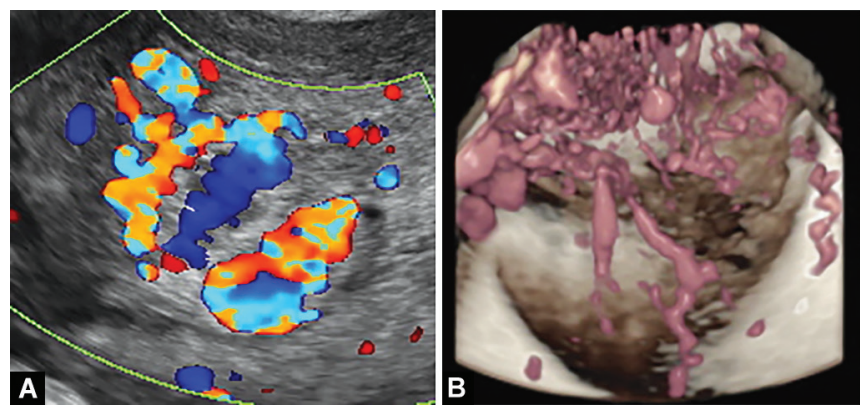

Figs 25A and B: (A) Homogeneous distribution of the blood vessels in the myometrial lesion on color Doppler; (B) Heterogeneous distribution of the blood vessels in the lesion on 3D power Doppler
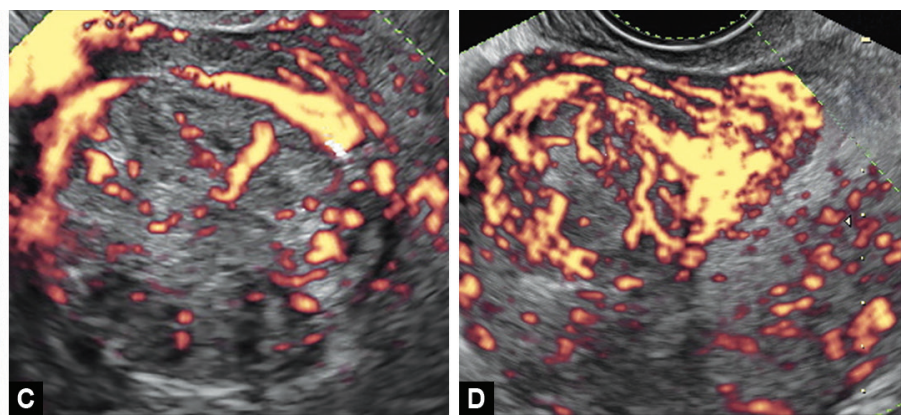

Figs 26A to D: Vascular score of 1-4, as: (A) Score 1—no color; (B) Score 2-minimal vascularity; (C) Score 3-moderate vascularity; (D) Score 4abundant vascularity
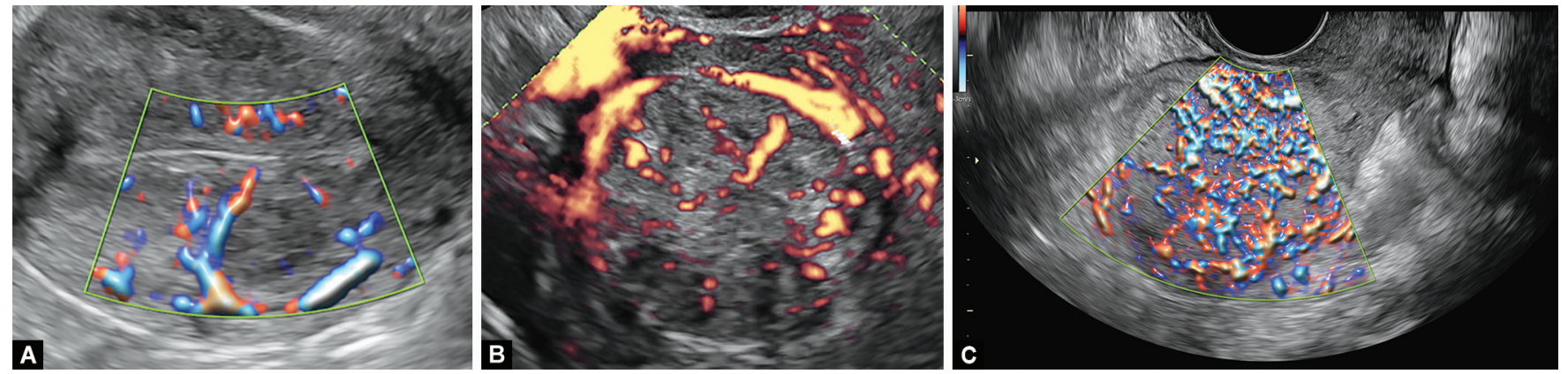

Figs 27A to C: (A) Circumferential vascularity in a fibroid as seen on high-definition (HD) flow; (B) Circumferential and intralesional vascularity in a fibroid as seen on power Doppler; (C) Translesional vascularity in adenomyosis as seen on HD flow 

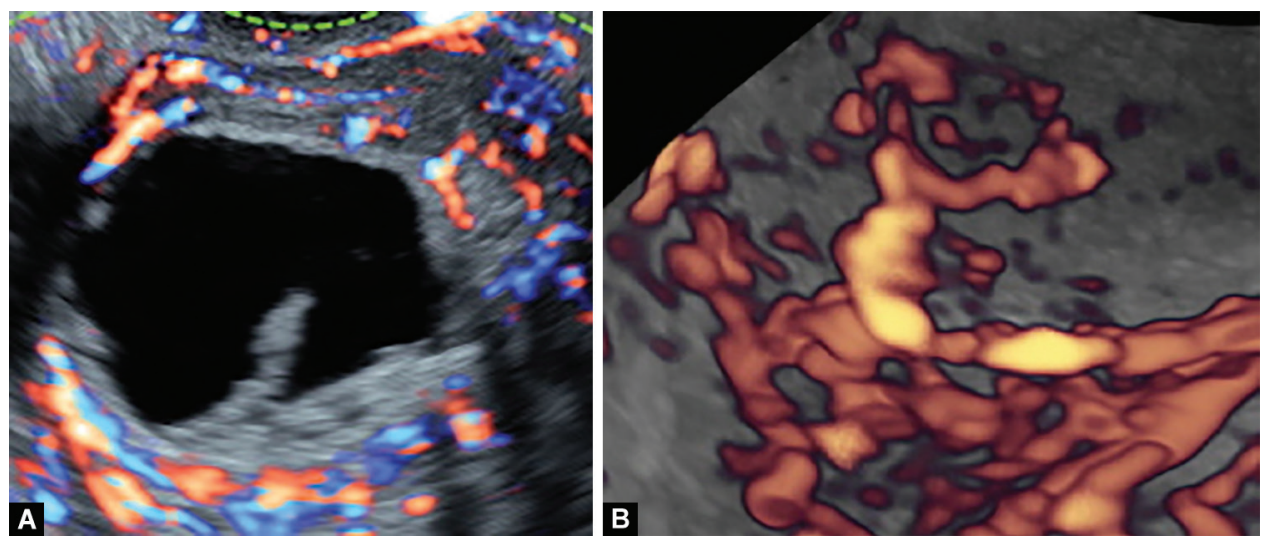

Figs 28A and B: (A) All vessels in the lesion showing almost equal diameter on HD flow; (B) Abnormal tortuous vessels, irregular caliber, when individual vessel shows variability in caliber as seen on power Doppler

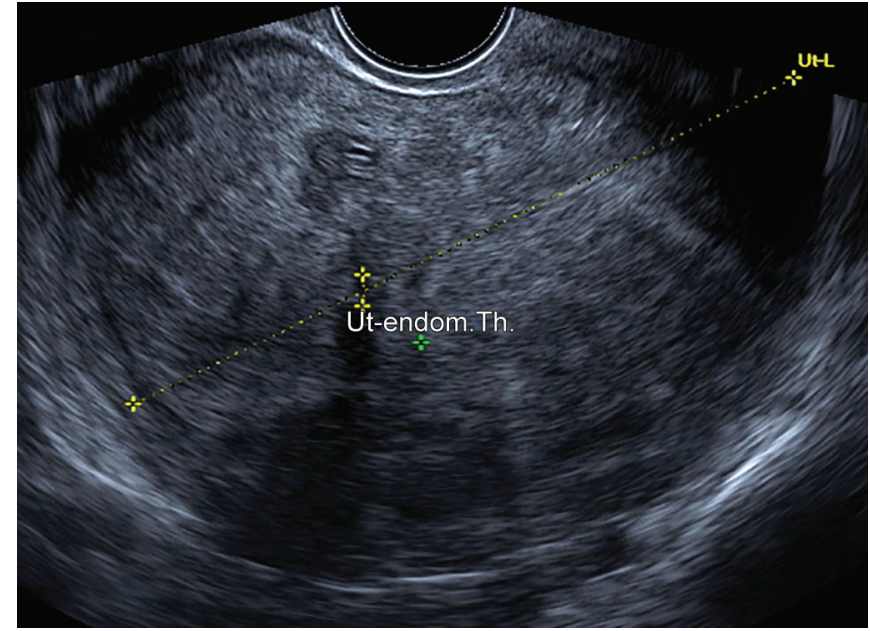

Fig. 29: Heterogeneous myometrial echogenicity of adenomyosis as seen on B-mode transvaginal ultrasound

Myometrial lesions commonly seen are fibroids, adenomyosis, adenomyomas, and leiomyosarcomas. Fibroids and adenomyosis are among the commonest pathologies found in patients presenting with various gynecological complaints and also with infertility.

\section{Adenomyosis}

Adenomyosis is an ectopic growth of endometrium into the myometrium and is common in multiparous women. But it is seen in women with a history of any obstetric event or any surgical procedure disturbing the integrity of the basal layer of endometrium. Pathologically, adenomyosis is defined as endometrial glands and stroma in the myometrium of half of one low-power field beneath the endomyometrial junction. These patients may present with menorrhagia, metrorrhagia, pelvic pain, dysmenorrhea, and dyspareunia. But adenomyosis is often an incidental finding on ultrasound. Patients with adenomyosis often present with infertility, and one of the major causes for this is inadequate endometrial receptivity. This may be due to physical compression effect due to thickened myometrium but may also be due to inadequate development of estrogen receptors due to altered vascularity. Pathologies, like leiomyomas (6-28\%),

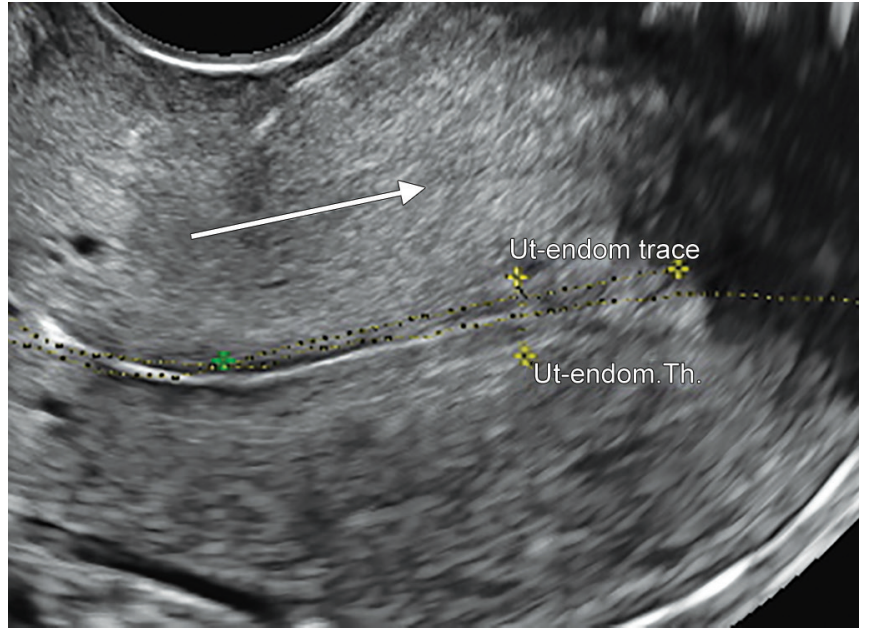

Fig. 30: Salt and pepper appearance of adenomyosis as seen on transvaginal B-mode ultrasound of the uterus, marked by arrow

and endometrial hyperplasia may coexist with adenomyosis. On clinical examination, the uterus feels bulky and is tender on palpation.

\section{On Ultrasound}

Adenomyosis may be diffuse and focal. ${ }^{3}$

Depending on the severity of the adenomyosis, the uterus may or may not appear bulky. It may cause symmetrical or asymmetrical thickening of myometrium. The affected myometrium shows a coarse heterogeneous echogenicity. Typically, the myometrium shows alteration in the echogenicity depending on the phase of the menstrual cycle. Heterogeneous echogenicity has a sensitivity of $88 \%$ for diagnosis of adenomyosis ${ }^{3}$ (Fig. 29).

The endometrial tissue that proliferates inside the myometrium tends to bleed on progesterone withdrawal during menstrual cycle. This gives the uterus a typically speckled appearance resembling "salt and pepper" (hyperechoic areas and hypoechoic areas) ${ }^{4}$ (Fig. 30). Adenomyosis being an invasive lesion has indistinct margins.

Adenomyosis presents with alternate vertical hyper- and hypoechoic zones, typically described as "rain in forest" or a "vanishing blind" appearance of the myometrium. This is described in MUSA as fan-shaped acoustic shadowing and is typical of 


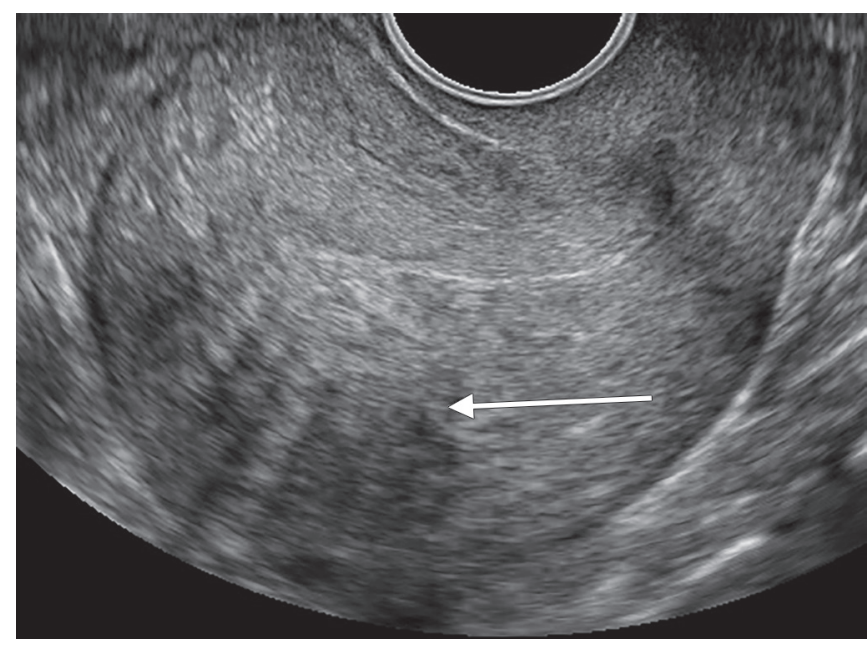

Fig. 31: Rain in forest or vanishing blind appearance of adenomyosis as seen on transvaginal B-mode ultrasound of the uterus, marked by arrow

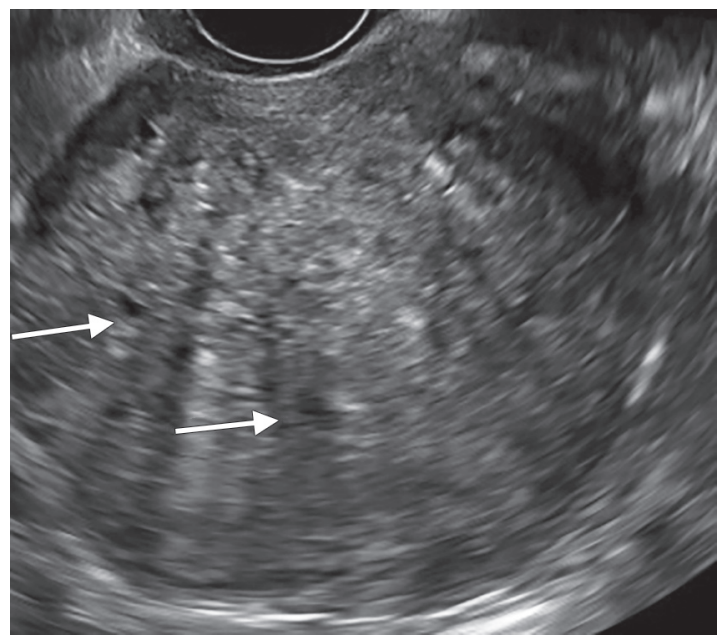

Fig. 33: Myometrial cysts (as marked by arrows) are seen in the heterogeneous adenomyotic myometrium

adenomyosis (Figs 31 and 32). Anechoic irregular cystic spaces of 5-7 mm in diameter will disrupt the homogeneous echo pattern of the myometrium (Fig. 33). These are myometrial cysts. Myometrial cysts have $98 \%$ specificity and $78 \%$ accuracy for diagnosis of adenomyosis. The myometrial cysts typically have hyperechoic margins. This probably is the active endometrial tissue. Echogenic flecks seen at the endometriomyometrial junction are collapsed myometrial cysts. Hyperechoic areas in myometrium may be due to fresh bleed or collapsed endometrial cavities. In some cases, hyperechoic spots or areas are also seen in the subendometrial myometrium and these may be islands of active endometrial tissue. These may be named as endometrial buds.

Junctional zone is irregular or interrupted and in severe cases also inaccessible. High-resolution ultrasound now can show the endometrial strands extending into the myometrium, interrupting the JZ (Fig. 34).

Adenomyotic uteri may commonly show an abnormal curvature of the fundal endometrial cavity in a direction opposite to the normal direction of the fundus (Fig. 35). This is described as question mark sign of the uterus. ${ }^{5}$

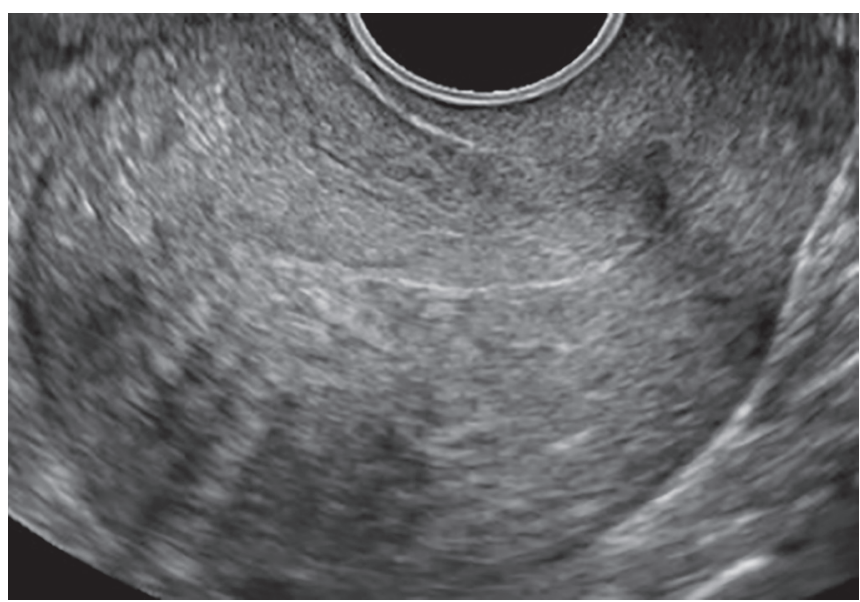

Fig. 32: Fan-shaped acoustic shadowing of adenomyosis as seen on transvaginal B-mode ultrasound of the uterus

The more difficult situation is the adenomyoma. This is a localized adenomyosis, with heterogeneous echogenicity and fairly defined margins, and so is difficult to differentiate from degenerated fibroid as this also has heterogeneous echogenicity. But the main differentiating point between the adenomyoma and the fibroid is the capsular vascularity that is seen in the fibroid but is never seen in adenomyoma (Fig. 36). Like generalized adenomyosis, it alters its echogenicity with the phase of the cycle, which is never the case in fibroid. Still one more important differentiating point is obliteration of the JZ in adenomyoma, unlike fibroid, and absence of edge shadows.

On color Doppler, affected myometrium shows markedly increased vascularity with vascular dilatation (Fig. 37). These vessels have resistance index $(\mathrm{RI})$ of $0.56 \pm 0.12$. Diameter of these vessels is larger than those of normal myometrium. ${ }^{6}$

Vascularity in adenomyosis is a penetrating type - translesional in distribution. Translesional vascularity is also seen in adenomyoma. Decreased uterine artery resistance and increased velocity are also noted. This is because adenomyomatous tissue demands more blood supply than the normal myometrial tissue.

Though sonohysterography is not a routine investigation for adenomyosis, if done by chance, it shows contrast (negative or positive), percolating into the myometrium.

Endometrial strands extending into myometrium are also seen on 3D ultrasound. When seen on 3D power Doppler also a vascular fibroid shows circumferentially arranged blood vessels, while in adenomyoma the vascular arrangement is radial or penetrating (Fig. 38). It is the JZ assessment that was not adequately possible with B-mode ultrasound always. Therefore, magnetic resonance imaging (MRI) was considered as an imaging modality for adenomyosis. Difference in the JZ thickness at two different places around the endometrium of more than $4 \mathrm{~mm}$ or a JZ thickness of $12 \mathrm{~mm}$ has $88 \%$ sensitivity and $85 \%$ accuracy for the diagnosis of adenomyosis. ${ }^{7}$ Three-dimensional transvaginal sonography (TVS) markers: ${ }^{7,8} \mathrm{JZ}$ difference of more than $4 \mathrm{~mm}$ and JZ infiltration and distortion have been proved as highly sensitive markers for the diagnosis of adenomyosis (Fig. 39).

Elastography is a modality of ultrasound that measures tissue strain. This is useful for differentiating myometrial lesions, though it is more commonly used for differentiating benign from malignant lesions in breast, liver, and other organs. Elastography may be qualitative or quantitative. Qualitative elastography subjectively compares the stiffness/firmness of various tissues, whereas quantitative elastography may measure the firmness objectively. 

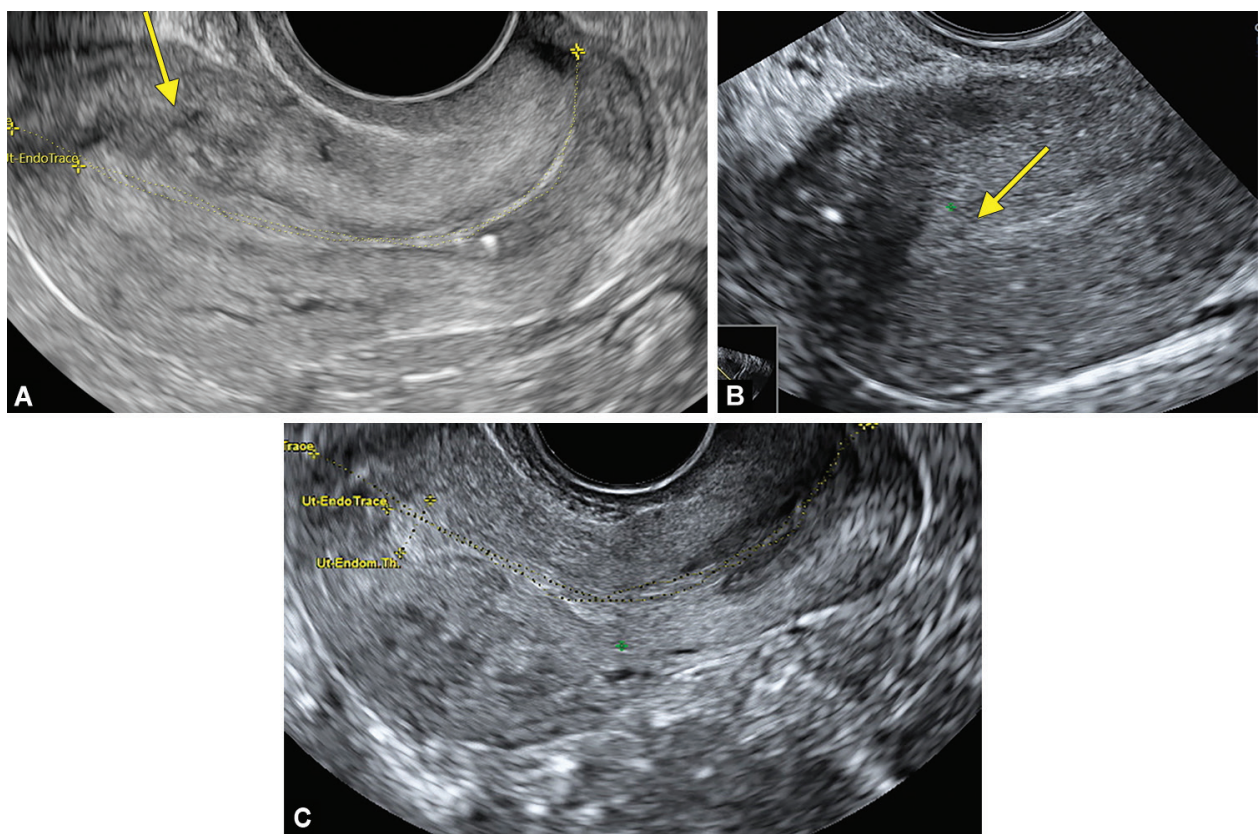

Figs 34A to C: (A) Transvaginal ultrasound B mode showing anteverted anteflexed uterus, midsagittal section of the uterus with irregular endometriomyometrial junction on the anterior aspect (arrow); (B) Transvaginal ultrasound B mode showing anteverted anteflexed uterus, midsagittal section of the uterus with interrupted endometriomyometrial junction on the anterior aspect due to endometrial strands extending into myometrium (arrow); (C) Transvaginal ultrasound B mode showing anteverted anteflexed uterus, midsagittal section of the uterus with inaccessible endometriomyometrial junction

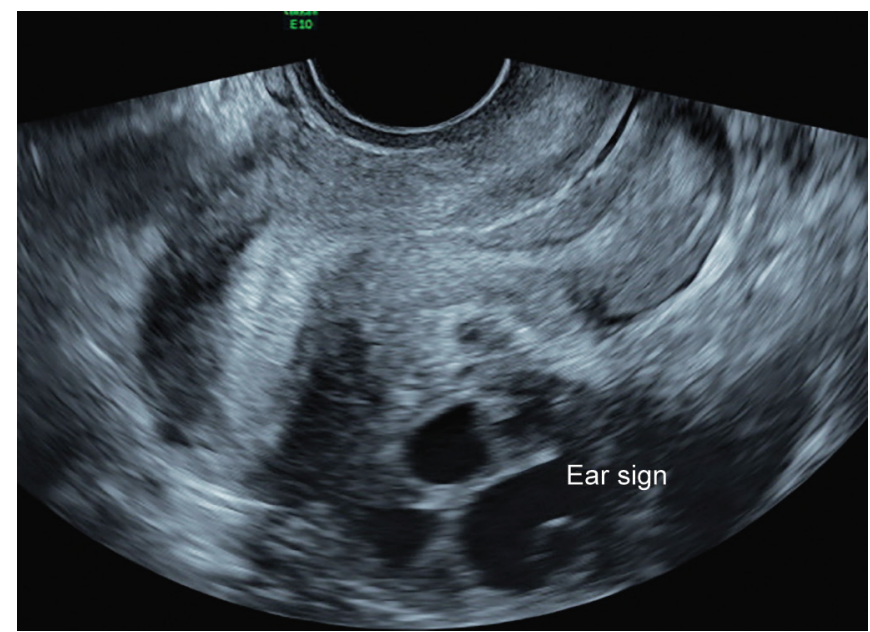

Fig. 35: Transvaginal ultrasound B mode showing anteverted anteflexed uterus, midsagittal section of the uterus with question mark sign

The tissues with different firmness are seen in different colors of the light spectrum. Blue side of this spectrum demonstrates hard or firm tissues, and red side of the spectrum demonstrates soft tissues, e.g., bowel appears red or yellow and myometrium shows uniform blue color. Fibroids are firmer (blue) than the myometrium, and adenomyosis is softer (green). Elastography-based diagnosis is in excellent agreement with $\mathrm{MRI}^{9}$ (Fig. 40).

Ultrasound features of adenomyosis

- Generalized/localized thickening of myometrial wall

- Asymmetrical thickening of anterior and posterior myometrial wall

- Heterogeneous myometrium: salt and pepper appearance
- "Rain in forest" or "vanishing blind" appearance/fan-shaped acoustic shadowing

- Echogenic flecks, lines, and buds

- Myometrial cysts

- Endometrial strands penetrating into the myometrium

- Question mark sign

- Irregularity and interruption of endometrio-myometrial JZ.

- Translesional vascularity

- Vessels with diameter larger than that of normal spiral vessels

- Abnormal orientation of myometrial vessels (chaotic arrangement)

\section{FIBROID}

Fibroids are found in $20-40 \%$ of women of more than 30 years of age and are more common in nulliparous. These are mostly an incidental finding but may sometimes present with dysmenorrhea, menorrhagia, infertility, and pregnancy-related problems. Fibroids affect fertility because of the following reasons:

- Distortion of endometrial cavity due to submucous fibroid

- Pressure of the fibroid and stretching of endometrium overlying it causing atrophy of endometrial glands and stroma

Buttram et al. have shown in their study that fertility is chiefly affected by submucous fibroids due to altered uterine contractility, deranged cytokine profile, abnormal vascularization, and chronic endometrial inflammation. ${ }^{10}$ These are benign tumors of myometrial and fibrous tissue origin, arising from the smooth muscle of uterine fundus and corpus. Fibroids are known to have a genetic basis and also have been shown to have a familial distribution or a hereditary transmission. Loss of tumor suppressor genes may be one of the causes. Estrogen and progesterone appear to promote the growth of fibroids as these have more estrogen and progesterone receptors 

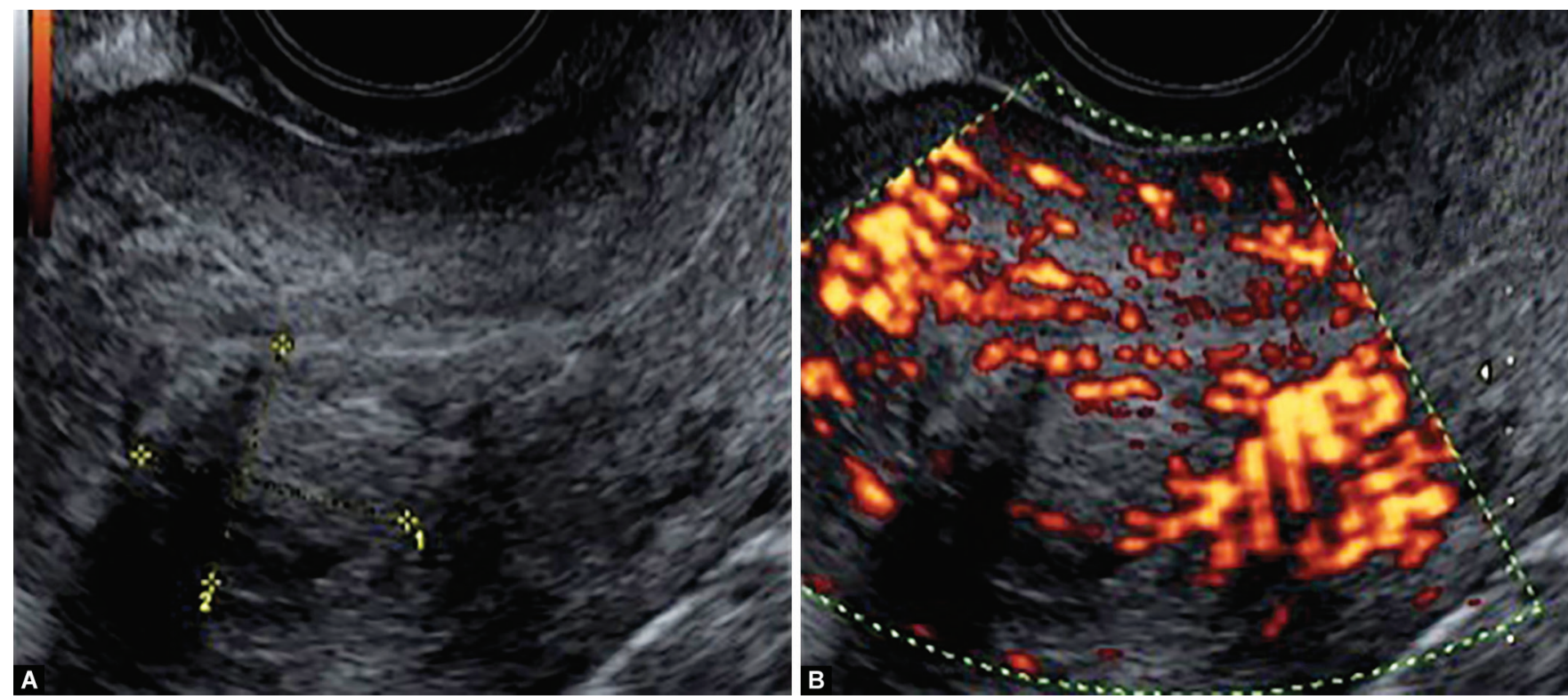

Figs 36A and B: Absence of circumferential vascularity and only intralesional vascularity in adenomyoma seen as a localized ill-defined lesion on B mode

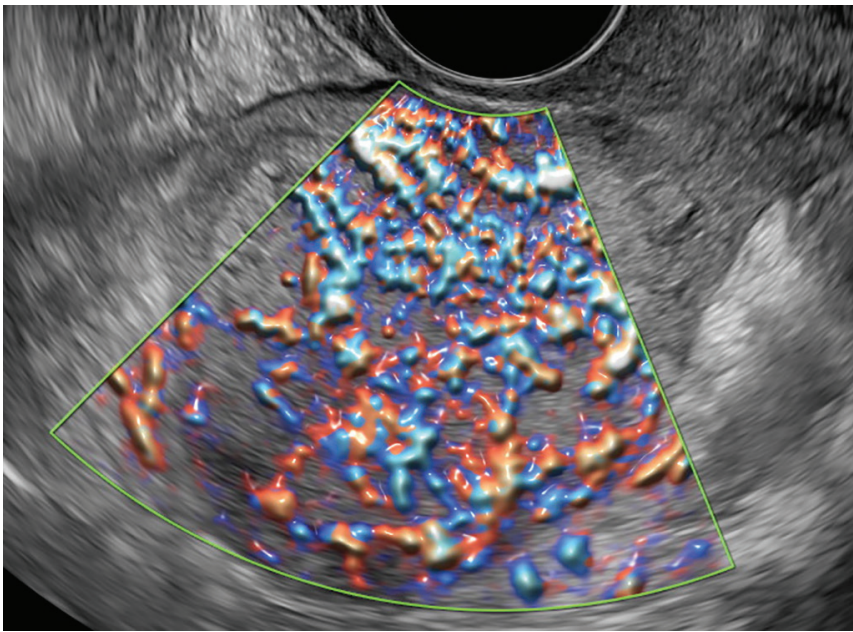

Fig. 37: HD flow showing translesional vascularity with nonparallel arrangement and increased diameter of the blood vessels (than the normal spiral vessels) in adenomyosis

as compared to normal myometrial tissue. Insulin-like growth factor may also affect the growth of the fibroid.

These may be seen in a variety of locations in the uterus. Depending on their location in relation to myometrium, endometrium or serosa, these are classified as types subendometrial and subserosal and intramural fibroids.

Subserosal and intramural fibroids were further classified as follows:

Type 0: pedunculated subserosal fibroid

Type 1: involvement of $<50 \%$ of the outer uterine wall

Type 2: $>50 \%$ of the myometrial wall

Type 3: fibroids extending from mucosa to serosa

Submucosal fibroids were further classified as follows: ${ }^{11}$

Type 0 , if they are pedunculated and $100 \%$ in the cavity (Fig. 41A)

Type 1 , if the fibroid is $>50 \%$ in cavity (Fig. 41B)

Type 2 , if less than $50 \%$ of the fibroid is protruding in the cavity (Fig. 41C).
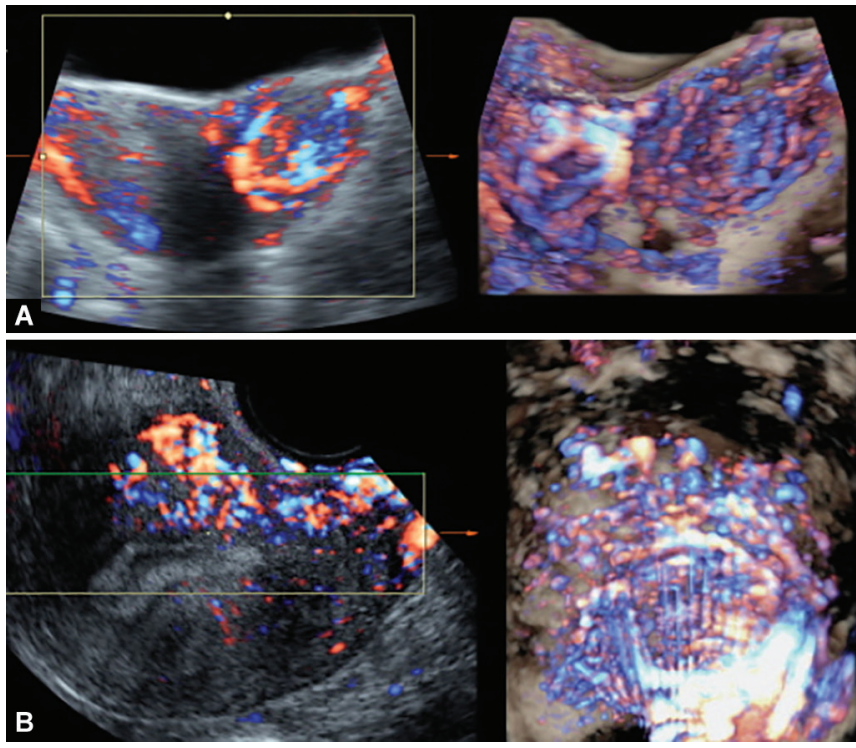

Figs 38A and B: (A) 3D power Doppler showing vascular fibroid with circumferentially arranged blood vessels; (B) Adenomyoma showing penetrating vascular pattern

According to the new Federation of International Societies of Gynecologists and Obstetricians (FIGO) classification, fibroids are classified into eight types ${ }^{2}$ (Fig. 42).

\section{On Ultrasound}

On ultrasound, fibroid appears as a well-defined, hypoechoic, homogeneous, round/oval, solid lesions, with peripheral hypoechoic rim due to displacement of myometrial fibers (Fig. 43). When large and especially subserosal or intramural they distort the serosal surface, whereas when subendometrial they distort the endometrial cavity. Fibroids typically show edge shadows along with the linear stripes like fan-shaped acoustic shadowing (Fig. 44). Echogenicity increases with increasing amount of fibrous tissue and vascularity. 
Degeneration in fibroids lead to heterogenicity of texture and these may become hyperechoic (Fig. 45). Cystic degeneration leads to anechoic spaces in the fibroid (Fig. 46). Red degeneration is the most common form of degeneration in fibroids with pregnancy, though red degeneration is not confidently diagnosed on ultrasound. When the fibroid undergoes an acute degeneration, patient may present with marked pain, sometimes vomiting and fever and the fibroid shows increase in size. Calcification may be seen in fibroids as hyperechoic $(++)$ areas with posterior shadowing (Fig. 47). When uterine artery embolization is done for fibroids, air may be seen in the fibroids as hyperechoic areas with comet shadows.

Correct localization of the fibroid on imaging is essential for better planning of the surgery. That is why fibroid mapping is an essential part of reporting a fibroid. This is especially important when there are multiple fibroids. Mapping can be correctly done by survey of the uterus in longitudinal and transverse axes by sweeping across the entire uterus in sagittal and transverse planes on $\mathrm{B}$ mode and also by the use of $3 \mathrm{D}$ for assessing the correct location on the coronal plane. In cases with multiple fibroids, when the endometrium is also grossly distorted, establishing the correct location of these fibroids may be difficult and then the endometrial line may be traced from the cervical canal to cephalad.

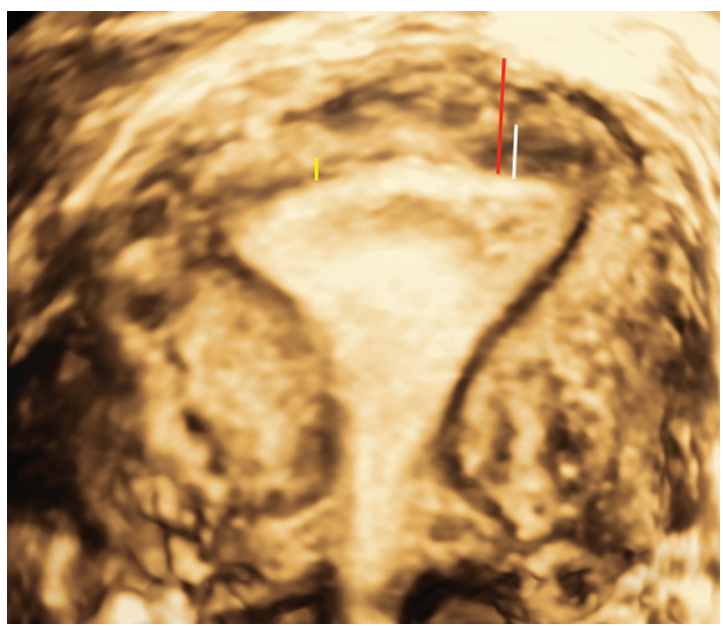

Fig. 39: Irregularity of the JZ in adenomyosis as seen on 3D ultrasound with the white line showing $J_{\max }$ and yellow line showing $J_{\min }$
The correct location of the fibroid can be defined by:

- Its position in the uterus - involving upper, middle, or lower part of the body of the uterus or the cervix

- Involving anterior, posterior, or right/left lateral walls

- Its position/penetration according to the FIGO classification

- Its size-preferably three orthogonal diameters

- Its distance from the endometrium or from the serosa to which ever wall it is closer.

\section{On Doppler}

Peripheral vascularity is typical of a fibroid (Fig. 27A). These vessels are vessels of displaced muscle fibers and therefore show a RI of $0.54 \pm 0.08,{ }^{12}$ similar to that of normal myometrial vessels. Resistance is higher in submucosal fibroids and deep intramural fibroids compared to subserosal fibroids but lower compared to submucosal fibroids. This variability is because of the compression effect of the muscle fibers on the fibroid and therefore on the surrounding vessels. Fibroids with degeneration show intralesional vascularity with low resistance flow and a vascular score of 2-3 (Fig. 27B). In addition to the peripheral vascularity, amount of vascularity decides the activity or growth potential of a fibroid. Malignant change leading to leiomyosarcoma shows a resistance as low as $0.37 \pm$ $0.03 \mathrm{RI}$, with rapid growth in size and disruption of well-defined margins (Fig. 48). But resistance indices of vessels cannot be used as only evidence for malignant change, because it is equally low in
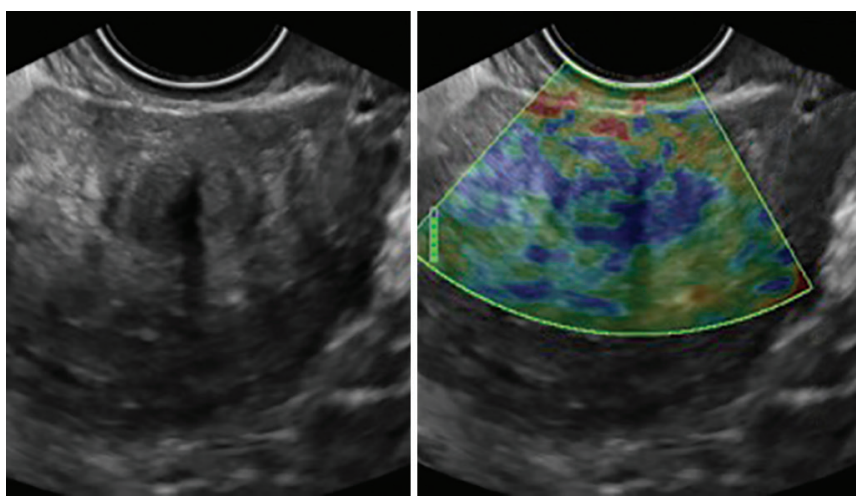

Fig. 40: Elastography showing blue-colored fibroid indicating its firm consistency
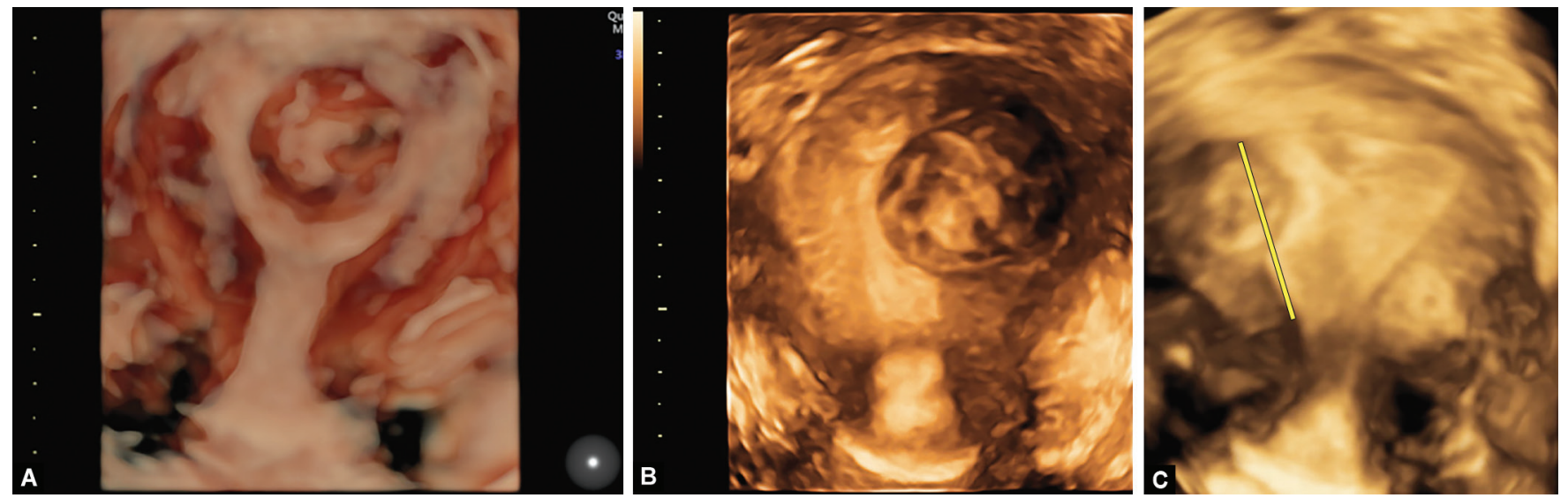

Figs 41A to C: Submucous fibroids, type 0, 1, and 2 demonstrated on 3D ultrasound on coronal section in A, B, and C, respectively 


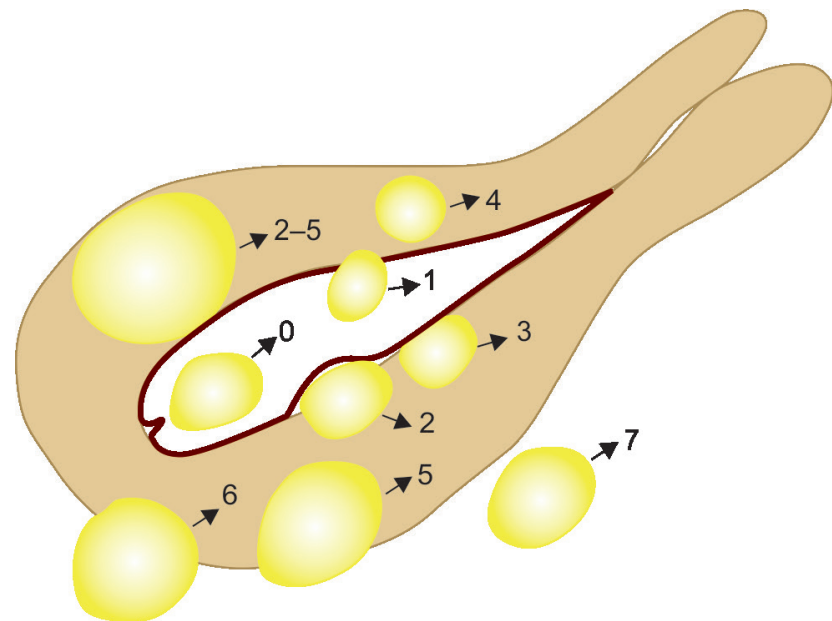

Fig. 42: Diagrammatic demonstration of types of fibroid according to FIGO classification

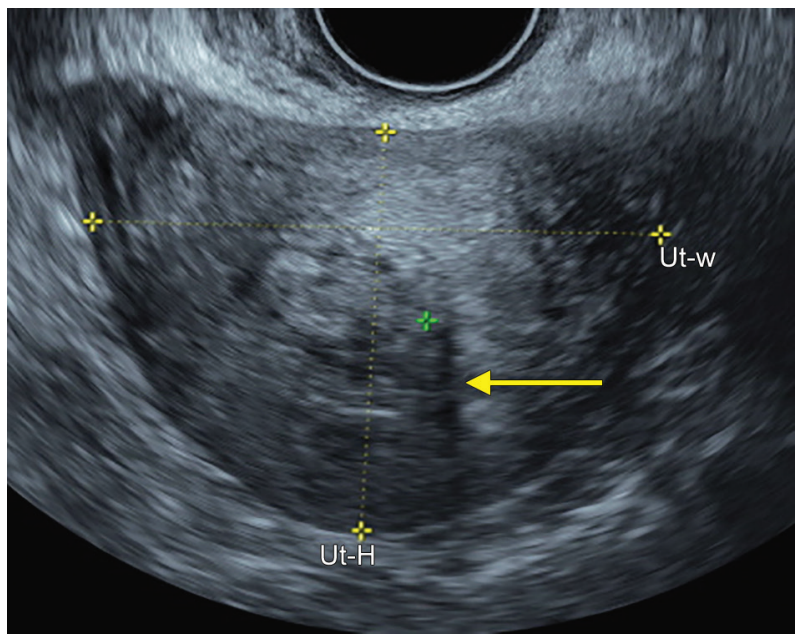

Fig. 44: Arrow showing edge shadow of fibroid on B-mode transvaginal ultrasound

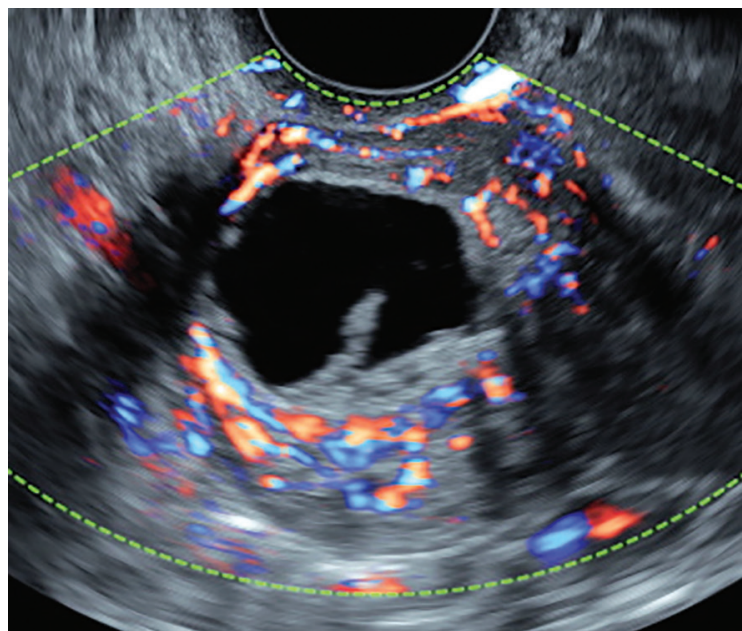

Fig. 46: Cystic degeneration of the fibroid seen as anechoic center with HD flow showing circumferential flow

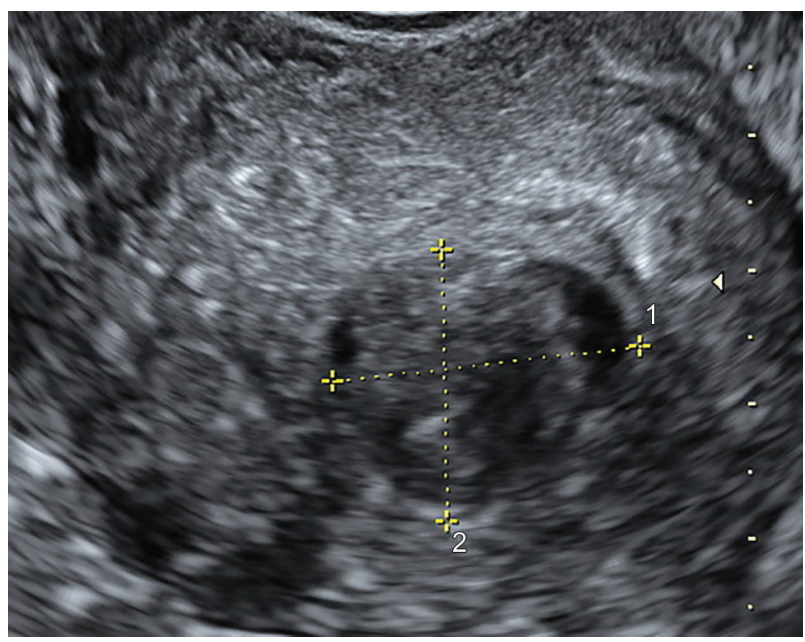

Fig. 43: Round well-defined isoechoic lesion is seen in the myometrium with hypoechoic margins - fibroid on B-mode transvaginal ultrasound

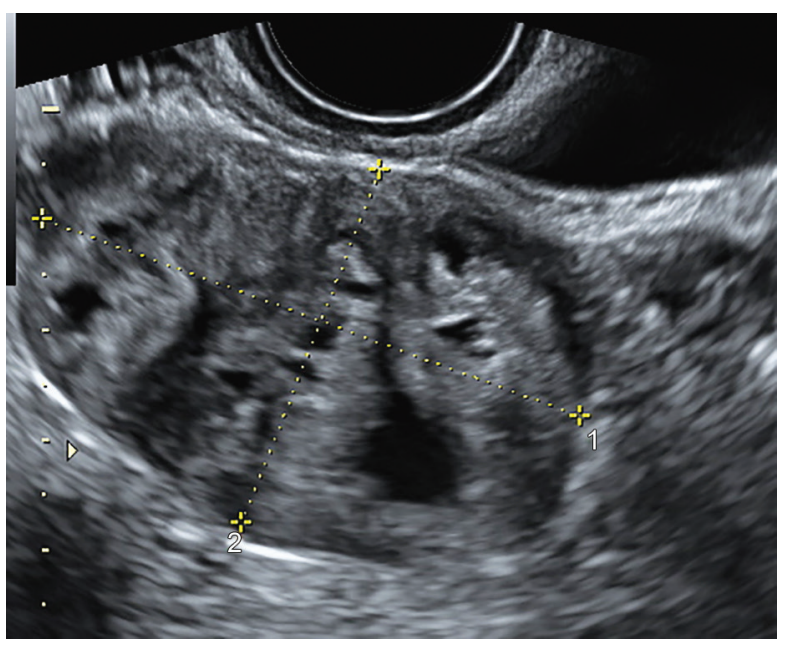

Fig. 45: Degenerated fibroid showing heterogeneous echogenicity

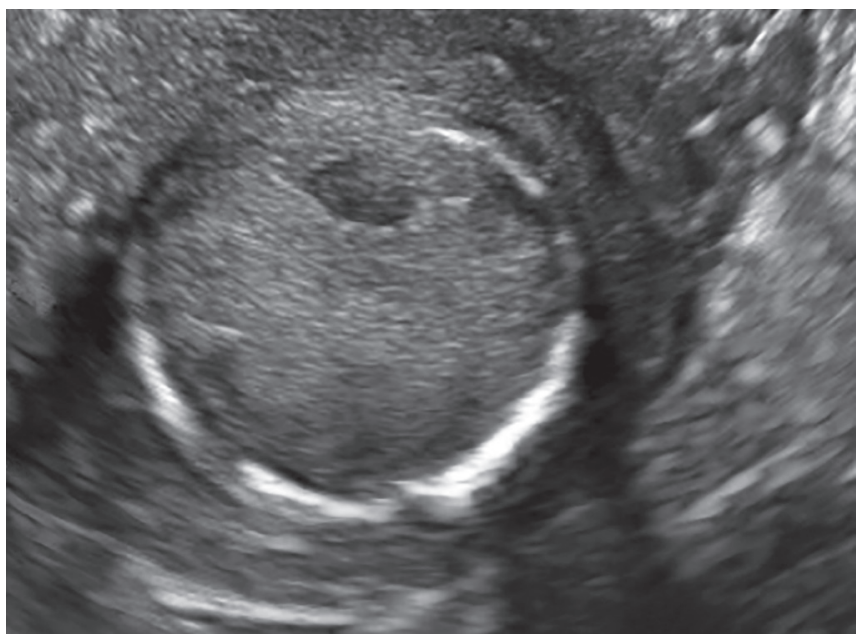

Fig. 47: B-mode transvaginal ultrasound showing fibroid with a calcified rim 


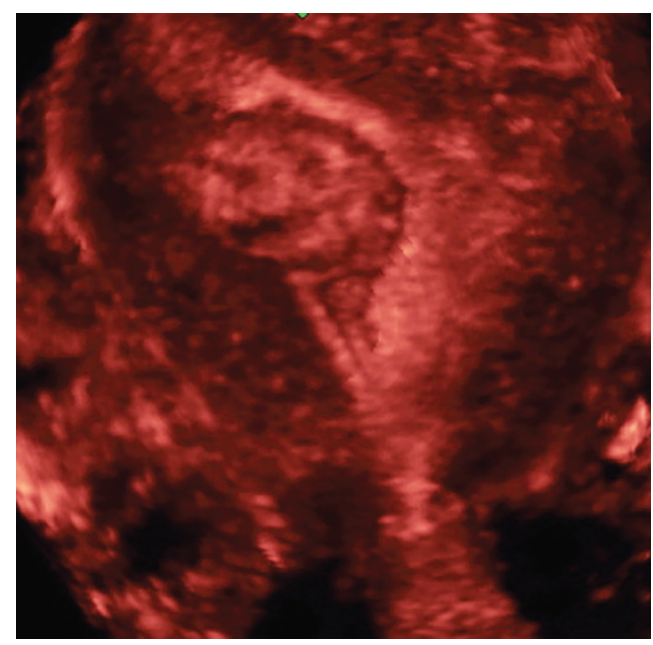

Fig. 48: 3D ultrasound of the uterus showing coronal section of the uterus, with a submucous fibroid showing ill-defined margin on its myometrial surface

degenerating fibroid also. In fact even on B-mode ultrasound due to the heterogenicity being a common finding in degenerating as well as malignant fibroid, it is not possible to confidently diagnose a leiomyosarcoma on B mode or color Doppler. Though when the $\mathrm{Rl}$ of the intralesional vessels is greater than 0.45 , along with a part of the fibroid margins being ill-defined, leiomyosarcoma may be suspected. Uteri with fibroids also show higher uterine artery peak velocity and lower RI (mean: $0.74 \pm 0.09)^{12}$

\section{Volume Ultrasound}

Volume ultrasound is especially useful in fibroids to find the relationship of fibroids to the endometrium and assess the distortion or invasion of the endometrium due to fibroid as it can evaluate the lesion in three orthogonal planes. Sonohysterography with saline combined with 3D ultrasound may demonstrate the distortion of the endometrial cavity by submucosal fibroids better. 13,14

3D power Doppler indices—VI, Fl, and VFI—are especially useful for follow-up of fibroids in patients who are on GnRha therapy, menopausal, or pregnant. In the former two, decreasing 3D power Doppler indices are seen against increasing $\mathrm{VI}, \mathrm{Fl}$, and $\mathrm{VFI}$ values are seen in fibroids in pregnancy.

Fibroids reporting should include the following:

- Homogeneous/heterogeneous

- Well-defined

- Number

- Location, size, site, penetration

- Mapping

- Vascularity_circumferential, equal diameter, homogeneous/ heterogeneous distribution

- Red, hyaline, cystic/myxoid, or hydropic degeneration

- It is important to have a standard report format for any scan that first describes the ultrasound features of a lesion/organ and then derives the diagnosis

Diffuse leiomyomatosis is a rare condition in which there is diffuse and uniform involvement of entire myometrium by multiple fibroids.

Fibroids are also seen arising from the cervix and show similar appearances as fibroids in the uterus (Fig. 49).

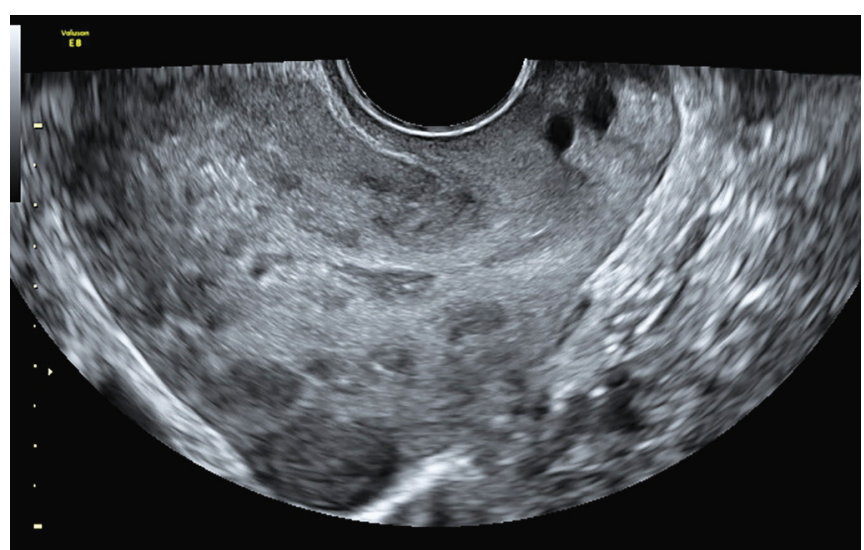

Fig. 49: Multiple fibroids (myomas) spread across the entire uterusleiomyomatosis

\section{Disseminated Peritoneal Leiomyomatosis (DPL)}

This is a very rare condition. There are several nodules of smooth muscle seen scattered throughout the peritoneum. It may occur due to seeding during surgeries, especially laparoscopic morcellation. Multiple lesions may distort the pelvic anatomy and therefore lead to symptoms but may also occur spontaneously at times. It is associated with elevated estrogen levels. According to another hypothesis, it is also thought to be due to metaplasia of subperitoneal mesenchymal cells. ${ }^{15}$ Diameter of the lesions may vary from 0.1 to $10 \mathrm{~cm}$. These lesions appear to have estrogen and progesterone receptors and also luteinizing hormone (LH) receptors. ${ }^{16,17}$

\section{Benign Metastasizing Leiomyoma}

In the uterus it appears like a typical fibroid, but similar lesion may be seen in the lungs and may lead to deterioration of lung function. Oophorectomy may treat the condition. Lung lesions are also seen in another fibroid-like condition called lymphangio-leiomyomatosis and this is more serious condition and has a genetic origin.

\section{Conclusion}

The most common myometrial lesions are adenomyosis and fibroid. Though on B-mode ultrasound, these have certain overlapping features, Doppler is diagnostic for these, due to typical translesional, intralesional, and peripheral vascularity, respectively.

\section{References}

1. Van den Bosch T, Dueholm M, et al. Terms, definitions and measurements to describe sonographic features of myometrium and uterine masses: a consensus opinion from the Morphological Uterus Sonographic Assessment (MUSA) group. Ultrasound Obstet Gynecol Sept 2015;46(5):284-298. DOI: 10.1002/uog.14806.

2. Munro MG, Critchley HOD, et al. FIGO classification system (PALMCOEIN) for causes of abnormal uterine bleeding in nongravid women of reproductive age. Int J Gynaecol Obstet 2011;113:3-13. DOI: 10.1016/j.ijgo.2010.11.011.

3. Raine-Fenning N, Jayaprakasan K, et al. Three-dimensional ultrasonographic characteristics of endometriomata. Ultrasound Obstet Gynecol 2008;31:718-724. DOI: 10.1002/uog.5380.

4. Aleem F, Pennisi J, et al. The role of color Doppler in diagnosis of endometriomas. Ultrasound Obstet Gynecol 1995;5:51-54. DOI: 10.1046/j.1469-0705.1995.05010051.x. 
5. Di Donato N, Bertoldo V, et al. Question mark form of uterus: a simple sonographic sign associated with presence of adenomyosis. Ultrasound Obstet Gynecol 2015;46(1):126-128. DOI: 10.1002/ uog. 14750.

6. Perrot N, Frey I, et al. Picture of the month. Adenomyosis: power Doppler findings. Ultrasound Obstet Gynecol 2001;17(2):177-178. DOI: 10.1046/j.1469-0705.2001.00378.x.

7. Exacoustos $C$, Brienza $L$, et al. Adenomyosis: three-dimensional sonographic findings of the junctional zone and correlation with histology. Ultrasound Obstet Gynecol 2011;37:471-479. DOI: 10.1002/ uog.8900.

8. Kupesic S, Kurjak A, et al. The value of transvaginal colour Doppler in the assessment of pelvic inflammatory disease. Ultrasound Med Biol 1995;21960:733-738. DOI: 10.1016/0301-5629(95)00013-H.

9. Stoelinga B, Hehenkamp WJK, et al. Real-time Elastography for assessment of uterine disorders. Ultrasound Obstet Gynecol 2014;43:218-226. DOI: 10.1002/uog.12519.

10. Buttram Jr VC, Reiter R. Uterine leiomyomata: etiology, symptomatology and management. Fertil Steril 1981;36:433-445. DOI: 10.1016/S0015-0282(16)45789-4; Taylor E, Gomel V. The uterus and fertility. Fertil Steril 2008;89:1-16. DOI: 10.1016/ j.fertnstert.2007.09.069.
11. Wamsteker K, de Blok S. Resection of intrauterine fibroids. In: Lewis BV, Magos AL. ed. Endometrial Ablation. Edinburgh, UK: Churchill Livingstone; 1993.

12. Kurjak A, Kupesic $S$, et al. The assessment of benign uterine tumour vascularization by transvaginal colour Doppler. Ultrasound Med Biol 1992;18:645-649. DOI: 10.1016/0301-5629(92)90079-P.

13. Balen $\mathrm{FG}$, Allen CM, et al. 3 dimensional reconstruction of ultrasound images of the uterine cavity. Br J Radiol 1993;66(787):588-591. DOI: 10.1259/0007-1285-66-787-588.

14. Weinraub Z, Maymon $\mathrm{R}$, et al. Three dimensional saline contrast hysterosonography and surface rendering of uterine cavity pathology. Ultrasound Obstet Gynecol 1996;8(4):277-282. DOI: 10.1046/j.1469-0705.1996.08040277.x.

15. Drake A, Dhundee J, et al. Disseminated leiomyomatosis peritonealis in association with oestrogen secreting ovarian fibrothecoma. BJOG 2001 Jun;108(6):661-664. DOI: 10.1111/j.1471-0528.2001.00132.x.

16. Akkersdjik GJ, Fli PK, et al. Malignant Lieomyomatosis peritonealis disseminata. Am J Obstet Gynecol 1990;163(2):591-593. DOI: 10.1016/0002-9378(90)91205-Q.

17. Butnor $\mathrm{KJ}$, Burchette $\mathrm{J}$, et al. Progesterone receptor activity in leiomyomatosis peritonealis disseminate. Int J Gynecol Pathol 1999;18(3):259-264. 